\title{
Interplay of ferromagnetism and triplet superconductivity in a Josephson junction
}

\author{
P. M. R. Brydon, ${ }^{1}$ Boris Kastening, ${ }^{2}$ Dirk K. Morr, ${ }^{3}$ and Dirk Manske ${ }^{1}$ \\ ${ }^{1}$ Max-Planck-Institut für Festkörperforschung, Heisenbergstrasse 1, 70569 Stuttgart, Germany \\ ${ }^{2}$ Institut für Theoretische Physik, Technische Hochschule Aachen, Physikzentrum, 52056 Aachen, Germany \\ ${ }^{3}$ Department of Physics, University of Illinois at Chicago, Chicago, Illinois 60607, USA
}

(Received 17 September 2007; published 4 March 2008)

\begin{abstract}
In this work we extend our earlier analysis of the novel Josephson effect in triplet superconductorferromagnet-triplet superconductor (TFT) junctions [Kastening et al., Phys. Rev. Lett. 96, 047009 (2006)]. In our more general formulation of the TFT junction, we allow for potential scattering at the barrier and an arbitrary orientation of the ferromagnetic moment. Several new effects are found upon the inclusion of these extra terms: for example, we find that a Josephson current can flow even when there is vanishing phase difference between the superconducting condensates on either side of the barrier. The critical current for a barrier with magnetization parallel to the interface is calculated as a function of the junction parameters, and is found to display strong nonanalyticities. Furthermore, the Josephson current switches identified in our previous work are found to be robust features of the junction, while the unconventional temperature dependence of the current is very sensitive to the extra terms in the barrier Hamiltonian.
\end{abstract}

DOI: 10.1103/PhysRevB.77.104504

PACS number(s): 74.50.+r, 74.45.+c, 74.78.-w

\section{INTRODUCTION}

A fundamental aspect of the superconducting state is the orbital symmetry of the order parameter, which is often regarded as a key indicator of the physical mechanisms underlying the pairing. Although the high- $T_{c}$ cuprates are undoubtedly the best-known example of a class of superconductors with unconventional (i.e., not $s$-wave) order parameter symmetry, ${ }^{1}$ this is also believed to be a feature of many heavy-fermion and organic superconductors. ${ }^{2}$ Of particular interest is the case of an odd-parity ( $p$-wave, $f$-wave, etc.) orbital pairing state, as this implies that the Cooper pair is in a triplet spin state. This of course opens the possibility of exotic magnetic properties of such superconductors. $p$-wave superconductivity was first anticipated shortly after the development of conventional BCS theory; ${ }^{3}$ only in the last decade, however, have the first examples of spin triplet superconductors been discovered. The most promising candidates for triplet superconductors are $\mathrm{Sr}_{2} \mathrm{RuO}_{4},{ }^{4-7}$ and $\mathrm{UPt}_{3} ;{ }^{8}$ it has also been proposed for a number of other compounds, such as (TMTSF) ${ }_{2} \mathrm{PF}_{6},{ }^{9} \mathrm{UGe}_{2},{ }^{10}$ and URhGe. ${ }^{11}$ Unique among these materials is $\mathrm{Sr}_{2} \mathrm{RuO}_{4}$, as it is well known that its normal state can be described by Fermi liquid theory. ${ }^{5,12}$

It has long been known that bound states can form at the surfaces of superconductors or at their interfaces with other materials. ${ }^{13}$ In a junction where two superconductors are separated by a barrier of sufficiently small width, these surface states overlap forming so-called Andreev bound states: this is of particular relevance in the theory of ballistic transport through Josephson junctions, as the tunneling through the Andreev bound states dominates the low-temperature transport. ${ }^{14}$ Andreev bound states are also formed in junctions involving unconventional superconductors. ${ }^{15,16}$ The Andreev states are responsible for many of the unique features of the current through such junctions, due to their strong sensitivity to the pairing symmetry of the superconductors on either side of the junction: a well-known experimental consequence of this sensitivity is the low-temperature anomaly in the Josephson current between two $d$-wave superconductors. ${ }^{16,17}$ There has recently been much interest in studying Josephson junctions involving $p$-wave superconductors, as the current through the Andreev bound states is predicted to have unique characteristics which may be considered to be the signature of the $p$-wave pairing state. ${ }^{18-24}$ The use of such superconductors in Josephson junctions is therefore expected to produce new phase-sensitive devices.

The construction of novel Josephson junctions also extends to the choice of tunneling barrier between the two superconductors. In particular, there has been much interest in the theory of the Josephson effect between two conventional superconductors separated by complex heterostructures or magnetic materials. ${ }^{25-27}$ An excellent example of the unusual properties of the latter class of junctions is provided by the prediction ${ }^{26}$ and subsequent experimental verification ${ }^{27}$ that a sign change in the current as a function of the temperature is possible for a metallic ferromagnetic barrier. Reversal of the current across a ferromagnetic barrier with potential scattering is also predicted to occur, although the origin of this effect is fundamentally different: ${ }^{28,29}$ in the metallic case, the sign change is due to the temperature dependence of the decay and oscillation lengths of the superconducting order parameter within the barrier; in the case with potential scattering, the effect is produced by the temperature-dependent changes in the occupation of the Andreev states.

Even though they are expected to have an intimate connection, the interplay between magnetism and $p$-wave superconductivity remains poorly understood. A promising route of investigation into this fundamentally interesting problem is the fabrication of devices that combine these two phenomena in a controlled manner. ${ }^{20,23,24,30,31}$ In Ref. 24, the ballistic tunneling through a Josephson junction constructed by sandwiching a ferromagnet between two $p_{z}$-wave superconductors was studied, the so-called "triplet superconductorferromagnet-triplet superconductor" (TFT) junction. The Josephson current $\left(I_{J}\right)$ through the TFT junction demonstrated a very rich dependence upon the relative orientation 
of the $\boldsymbol{d}$ vectors of the two superconductors and the ferromagnetic moment of the tunneling barrier, which were all assumed to be parallel to the junction interface. As in the case of $s$-wave superconductors, the ferromagnetic barrier is responsible for a reversal of the current with increasing temperature. The authors also pointed out that in certain circumstances the current is very sensitive to the alignment of the junction components, and noted that this sensitivity could be exploited to create "current switches:" very small changes in the alignments can cause large increases in the magnitude of $I_{J}$, effectively tuning the junction between on and off states, or alternatively an abrupt reversal of the direction of current flow can be produced. This has particular relevance to the field of quantum information technology, as these two-leveltype effects open the way for the development of novel types of quantum bits. ${ }^{32}$ Of course, the dependence of the current on the relative orientation of the junction components would also act as an important test of the $p$-wave symmetry of the superconductors.

In this work we extend and elaborate upon the work presented in Ref. 24 by the inclusion of additional scattering terms in the barrier, in particular a potential scattering term and a coupling to a component of the magnetic moment normal to the barrier interface. Our first objective is to assess to what extent the current switch effect and the sign reversal of the Josephson current with increasing temperature are robust to the more general description of the barrier. We show that the current switch effect is quite robust, while the temperature-dependent sign reversal of the current is considerably more sensitive with respect to the inclusion of other scattering terms.

Our second aim is to study the emergence of novel phenomena arising from the inclusion of these additional barrier terms. Specifically, we predict three new effects at $T=0$ : (i) if the $\boldsymbol{d}$ vectors of the left and right superconductors are not aligned, it is possible to generate a nonzero Josephson current even for zero phase difference $\phi$ between the two superconducting condensates; (ii) for appropriately chosen barrier potentials and angle between the $\boldsymbol{d}$ vectors, there is no net current flowing through the Andreev bound states for a finite range of phase differences between the two condensates; and (iii) the presence of potential scattering terms in a magnetic barrier can substantially enhance the Josephson current flowing through it. We furthermore calculate the spin transport properties of the junction in the case when spin-flip scattering is absent from the barrier. We find that the $z$ component of the spin current flows even for $\phi=0$, so long as the $\boldsymbol{d}$ vectors of the two superconductors are not aligned. Finally, we examine the dependence of the critical current on the barrier parameters and the alignment of the $\boldsymbol{d}$ vectors. We present a prediction for the "phase diagram" of the TFT junction (as a function of the barrier parameters and the $\boldsymbol{d}$-vector alignment) in which the phases correspond to different locations of the critical current in the current vs phase relations of the junction, and phase boundaries are given by nonanalyticities in the critical current. Apart from the inherent theoretical interest, this would also make for an excellent experimental test of our knowledge of the TFT junction.

In the first part of our paper we introduce in detail the Hamiltonian description of the TFT junction (Sec. II A). This

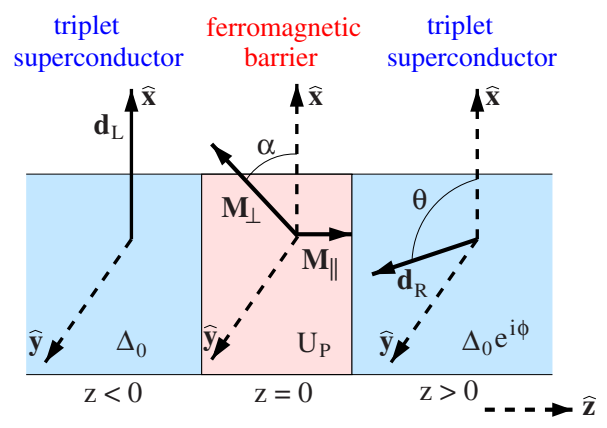

FIG. 1. (Color online) Schematic diagram of the TFT junction studied in this work. The figure shows the specific choice of parameters that we adopt from the end of Sec. II B onward.

is followed by the solution of the associated Bogoliubov-de Gennes equations in Sec. II B, where we obtain a general expression for the Andreev bound state energies $E_{a, b}$ and the Josephson current $I_{J}$. These results form the basis of Sec. III, where we discuss the dependence of $E_{a, b}$ and $I_{J}$ upon the several parameters characterizing the system. In Sec. III A we focus upon the case of aligned $\boldsymbol{d}$ vectors, including a discussion of the temperature dependence of the current in Sec. III A 1. This is followed in Sec. III B by the more general case of nonaligned $\boldsymbol{d}$ vectors. The critical current through the Josephson junction and the dependence of its first-order nonanalyticities upon the junction parameters are presented in Sec. III C. We conclude in Sec. IV with a summary of our results and an outlook for further work.

\section{THEORY}

\section{A. General Hamiltonian for a TFT junction}

We consider a one-dimensional Josephson junction oriented along the $z$ axis, constructed by sandwiching a ferromagnetic layer of width $d$ between two triplet superconductors (a schematic diagram of the TFT junction is provided in Fig. 1). For the triplet superconductors to the left and right of the junction, we assume that their respective $\boldsymbol{d}$ vectors $\boldsymbol{d}_{L}$ and $\boldsymbol{d}_{R}$ lie in the spin $x-y$ plane, and are parametrized by rotation angles $\theta_{L}$ and $\theta_{R}$ with respect to the $x$ axis. The magnetization of the barrier has a component $\boldsymbol{M}_{\perp}$ that lies in the $x-y$ plane and is parametrized by an angle $\alpha$ with respect to the $x$ axis, and also a component $\boldsymbol{M}_{\|}$in the $z$ direction. The barrier is also assumed to contain a potential scattering term.

The Josephson junction is described by the Hamiltonian $H=\int d z^{\prime} d z \mathcal{H}\left(z^{\prime}, z\right)$, where the Hamiltonian density is defined by

$$
\mathcal{H}\left(z^{\prime}, z\right)=\mathcal{H}_{0}\left(z^{\prime}, z\right)+\mathcal{H}_{\perp}\left(z^{\prime}, z\right)+\mathcal{H}_{\|}\left(z^{\prime}, z\right)+\mathcal{H}_{T}\left(z^{\prime}, z\right) .
$$

The first term in Eq. (1) describes the kinetic energy and the potential scattering by $\mathcal{U}_{P}(z)$,

$$
\mathcal{H}_{0}\left(z^{\prime}, z\right)=\sum_{\sigma} \psi_{\sigma}^{\dagger}\left(z^{\prime}\right) \delta\left(z^{\prime}-z\right)\left(-\frac{\hbar^{2} \partial_{z}^{2}}{2 m}-\mu+\mathcal{U}_{P}(z)\right) \psi_{\sigma}(z),
$$

where $\psi_{\sigma}(z)$ is the annihilation field operator for an electron of spin $\sigma$. In general, we can have different effective masses 
$m$ on either side of the junction and within the barrier itself, respectively, $m_{L}, m_{R}$, and $m_{B}$. This situation may be included in Eq. (2) by assuming a $z$-dependent $m$.

The interaction between the magnetic moment of the barrier and the spin of the conduction electrons is given in the standard way via

$$
\mathcal{H}_{\perp}\left(z^{\prime}, z\right)+\mathcal{H}_{\|}\left(z^{\prime}, z\right)=-\boldsymbol{M}\left(z^{\prime}, z\right) \cdot \sum_{\alpha, \beta} \psi_{\alpha}^{\dagger}\left(z^{\prime}\right) \boldsymbol{\sigma}_{\alpha \beta} \psi_{\beta}(z) .
$$

The term $\mathcal{H}_{\perp}$ describes the scattering of the quasiparticles by the magnetic moment in the $x-y$ plane. The orientation of the moment with respect to the $x$ axis is given by $\alpha$, and so we have the vectorial representation for the scattering potential $\boldsymbol{M}_{\perp}\left(z^{\prime}, z\right)=\delta\left(z-z^{\prime}\right) \mathcal{M}_{\perp}(z)(\cos \alpha, \sin \alpha, 0)$. We thus obtain

$$
\begin{aligned}
\mathcal{H}_{\perp}\left(z^{\prime}, z\right)= & -\delta\left(z-z^{\prime}\right) \mathcal{M}_{\perp}(z)\left[e^{-i \alpha} \psi_{\uparrow}^{\dagger}\left(z^{\prime}\right) \psi_{\downarrow}(z)\right. \\
& \left.-e^{i \alpha} \psi_{\uparrow}\left(z^{\prime}\right) \psi_{\downarrow}^{\dagger}(z)\right] .
\end{aligned}
$$

The scattering of the quasiparticle by the magnetization in the $z$ direction is described by the potential $\boldsymbol{M}_{\|}\left(z^{\prime}, z\right)$ $=\delta\left(z-z^{\prime}\right) \mathcal{M}_{\|}(z)(0,0,1)$, and so we have

$$
\mathcal{H}_{\|}\left(z^{\prime}, z\right)=-\delta\left(z-z^{\prime}\right) \mathcal{M}_{\|}(z)\left[\psi_{\uparrow}^{\dagger}\left(z^{\prime}\right) \psi_{\uparrow}(z)-\psi_{\downarrow}^{\dagger}\left(z^{\prime}\right) \psi_{\downarrow}(z)\right] .
$$

Finally, we have the triplet pairing term

$$
\begin{aligned}
\mathcal{H}_{T}\left(z^{\prime}, z\right)= & \Delta\left(z^{\prime}, z\right)\left(\psi_{\uparrow}^{\dagger}\left(z^{\prime}\right) \psi_{\downarrow}^{\dagger}\left(z^{\prime}\right)\right)(\boldsymbol{d} \cdot \boldsymbol{\sigma})\left(-i \sigma_{2}\right)\left(\begin{array}{l}
\psi_{\uparrow}^{\dagger}(z) \\
\psi_{\downarrow}^{\dagger}(z)
\end{array}\right) \\
& + \text { H.c. },
\end{aligned}
$$

with $\boldsymbol{d}$ vector $\boldsymbol{d}=\left(d_{x}, d_{y}, d_{z}\right)$. We choose real $\boldsymbol{d}$ vectors (so that they are "unitary," i.e., $\boldsymbol{d} \times \boldsymbol{d}^{*}=0$ ) that lie in the $x$-y plane, $\boldsymbol{d}=(\cos \theta, \sin \theta, 0)$. For this choice of $\boldsymbol{d}$ vector, the pairing term may be rewritten as

$$
\begin{aligned}
\mathcal{H}_{T}\left(z^{\prime}, z\right)= & \left\{\Delta\left(z^{\prime}, z\right)\left[e^{-i \theta} \psi_{\uparrow}^{\dagger}\left(z^{\prime}\right) \psi_{\uparrow}^{\dagger}(z)-e^{i \theta} \psi_{\downarrow}^{\dagger}\left(z^{\prime}\right) \psi_{\downarrow}^{\dagger}(z)\right]\right. \\
& \left.-\Delta^{*}\left(z^{\prime}, z\right)\left[e^{i \theta} \psi_{\uparrow}\left(z^{\prime}\right) \psi_{\uparrow}(z)-e^{-i \theta} \psi_{\downarrow}\left(z^{\prime}\right) \psi_{\downarrow}(z)\right]\right\} .
\end{aligned}
$$

For performing a Bogoliubov transformation it is convenient to introduce a matrix notation. We define the spinor

$$
\Psi(z)=\left(\psi_{\uparrow}(z), \psi_{\uparrow}^{\dagger}(z), \psi_{\downarrow}(z), \psi_{\downarrow}^{\dagger}(z)\right)^{T},
$$

which obeys the matrix anticommutation relation

$$
\left\{\Psi(z), \Psi^{\dagger}\left(z^{\prime}\right)\right\}=\delta\left(z-z^{\prime}\right) \hat{1} .
$$

We may then write the Hamiltonian in the form

$$
H=\frac{1}{2} \int d z^{\prime} d z \Psi^{\dagger}\left(z^{\prime}\right) \hat{\mathcal{H}}\left(z^{\prime}, z\right) \Psi(z) .
$$

The matrix Hamiltonian density $\hat{\mathcal{H}}\left(z^{\prime}, z\right)$ is defined by

$$
\hat{\mathcal{H}}\left(z^{\prime}, z\right)=\left(\begin{array}{cccc}
\delta_{z z^{\prime}}\left(T+\mathcal{U}_{P}-\mathcal{M}_{\|}-\mu\right) & e^{-i \theta} \Delta\left(z^{\prime}, z\right) & -\delta_{z z^{\prime}} \mathcal{M}_{\perp} e^{-i \alpha} & 0 \\
-e^{i \theta} \Delta^{*}\left(z^{\prime}, z\right) & -\delta_{z z^{\prime}}\left(T+\mathcal{U}_{P}-\mathcal{M}_{\|}-\mu\right) & 0 & \delta_{z z^{\prime}} \mathcal{M}_{\perp} e^{i \alpha} \\
-\delta_{z z^{\prime}} \mathcal{M}_{\perp} e^{i \alpha} & 0 & \delta_{z z^{\prime}}\left(T+\mathcal{U}_{P}+\mathcal{M}_{\|}-\mu\right) & -e^{i \theta} \Delta\left(z^{\prime}, z\right) \\
0 & \delta_{z z^{\prime}} \mathcal{M}_{\perp} e^{-i \alpha} & e^{-i \theta} \Delta^{*}\left(z^{\prime}, z\right) & -\delta_{z z^{\prime}}\left(T+\mathcal{U}_{P}+\mathcal{M}_{\|}-\mu\right)
\end{array}\right),
$$

where we have adopted the abbreviations $\delta_{z z^{\prime}} \equiv \delta\left(z-z^{\prime}\right)$, $T \equiv-\hbar^{2} \partial_{z}^{2} / 2 m, \mathcal{U}_{P} \equiv \mathcal{U}_{P}(z), \mathcal{M}_{\perp} \equiv \mathcal{M}_{\perp}(z)$, and $\mathcal{M}_{\|} \equiv \mathcal{M}_{\|}(z)$. We can easily see from Eq. (11) the justification for the separate parametrizations for the longitudinal and transverse components of the barrier magnetization: the former do not mix the spin states of the quasiparticles, whereas for the latter the $z$ component of spin is no longer a good quantum number. This has important consequences for the Andreev states and the Josephson current.

Up to now our discussion has been very general: in Eq. (11) the forms of the scattering potentials and the barrier width are left indeterminate. For the remainder of this work, however, we consider only the case where the barrier between the left and right superconductors is a point contact, and so we adopt the physically reasonable approximation that it is of infinitesimal width. ${ }^{14}$ We therefore replace the scattering potentials by $\delta$ functions:

$$
\mathcal{U}_{P}(z)=U_{P} \delta(z), \quad \mathcal{M}_{\perp}(z)=M_{\perp} \delta(z), \quad \mathcal{M}_{\|}(z)=M_{\|} \delta(z)
$$

Without loss of generality we choose $M_{\perp} \geqslant 0$.

For concreteness, we assume a gap with $p_{z}$ symmetry. In momentum space the superconducting gap therefore has the form

$$
\Delta(k)=\Delta_{T} \sin k a,
$$

where $a$ is the lattice constant of the system. It follows from Eq. (13) that in real space the gap takes the form

$$
\Delta\left(z^{\prime}, z\right)=-\frac{\Delta_{T}}{2 i a}\left[\delta\left(z^{\prime}-z+a\right)-\delta\left(z^{\prime}-z-a\right)\right] .
$$

Consider now a function $f(z)$ that is slowly varying on the length scale of the lattice. We may then approximate the integral as 


$$
\int d z^{\prime} \Delta\left(z^{\prime}, z\right) f\left(z^{\prime}\right)=\frac{\Delta_{T}}{2 i a}[f(z+a)-f(z-a)] \approx-i \Delta_{T} \partial_{z} f(z) .
$$

This approximation is valid in our Hamiltonian description so long as the Fermi vectors $k_{v}$ in the two superconductors satisfy $k_{\nu} a \ll 1$; the form Eq. (15) is also expected to give qualitatively correct results in the general case, in the same spirit as the free-electron description of the kinetic energy, Eq. (2). $\Delta_{T}$ is different on the left $(z<0)$ and right $(z>0)$ sides of the junction and given by $\Delta_{L} e^{i \phi_{L}}$ and $\Delta_{R} e^{i \phi_{R}}$, respectively, with $\Delta_{L, R}$ real and positive. Since only the phase difference between the left and right superconductors is of physical significance, we set $\phi_{L}=0$ and $\phi_{R}=\phi$ without loss of generality.

\section{B. Solution of the Bogoliubov-de Gennes equations}

We diagonalize the Hamiltonian Eq. (11) by performing a Bogoliubov-de Gennes (BdG) transformation. A detailed derivation of the $\mathrm{BdG}$ eigenequations is given in Appendix A; for the general Hamiltonian, the wave function $\Phi(z)$ and energy $E$ of the Andreev bound states is given by the solution to

$$
\int d z^{\prime} \hat{\mathcal{H}}\left(z, z^{\prime}\right) \Phi\left(z^{\prime}\right)=E \Phi(z)
$$

Under our assumption of $\delta$-function barrier potentials, and using the result Eq. (15), the Hamiltonian density is a function of a single variable. Equation (16) then simplifies to

$$
\hat{H} \Phi(z)=E \Phi(z),
$$

where

$$
\hat{H}=\left(\begin{array}{cccc}
T+\left(U_{P}-M_{\|}\right) \delta(z)-\mu & i e^{-i \theta} \Delta_{T} \partial_{z} & -e^{-i \alpha} M_{\perp} \delta(z) & 0 \\
i e^{i \theta} \Delta_{T}^{*} \partial_{z} & -T-\left(U_{P}-M_{\|}\right) \delta(z)+\mu & 0 & e^{i \alpha} M_{\perp} \delta(z) \\
-e^{i \alpha} M_{\perp} \delta(z) & 0 & T+\left(U_{P}+M_{\|}\right) \delta(z)-\mu & -i e^{i \theta} \Delta_{T} \partial_{z} \\
0 & e^{-i \alpha} M_{\perp} \delta(z) & -i e^{-i \theta} \Delta_{T}^{*} \partial_{z} & -T-\left(U_{P}+M_{\|}\right) \delta(z)+\mu
\end{array}\right),
$$

and $T$ is defined as for Eq. (11). $\Phi(z)$ satisfies two boundary conditions at the interface. If $\Phi_{\nu}(z)$ is a solution of Eq. (17) in the $\nu=L, R$ superconductor, the first boundary condition is given by the continuity of the wave function at the junction,

$$
\Phi_{L}(0)=\Phi_{R}(0) .
$$

In order to obtain the second boundary condition, we integrate the BdG equations across the junction barrier at $z=0$ by applying the operator $\int_{-\delta}^{+\delta} d z$ and subsequently letting $\delta \rightarrow 0$. The only contributions come from singular terms, which are the second derivatives of the components of $\Phi_{\nu}$ and the $\delta$ functions modeling the barrier itself. We hence obtain the condition

$$
\partial_{z} \Phi_{R}(0)-\partial_{z} \Phi_{L}(0)=2 \sqrt{k_{L} k_{R}}\left(\begin{array}{cc}
\left(Z-g^{\prime}\right) \hat{\sigma}_{0} & -g\left(\hat{\sigma}_{0} \cos \alpha-i \hat{\sigma}_{3} \sin \alpha\right) \\
-g\left(\hat{\sigma}_{0} \cos \alpha+i \hat{\sigma}_{3} \sin \alpha\right) & \left(Z+g^{\prime}\right) \hat{\sigma}_{0}
\end{array}\right) \Phi_{R}(0),
$$

where $k_{\nu}$ is the Fermi momentum on the $\nu$ side of the junction, defined in terms of the chemical potential $\mu_{\nu}$ $=\hbar^{2} k_{\nu}^{2} / 2 m_{\nu}$, and the dimensionless couplings $Z, g$, and $g^{\prime}$ are defined by

$$
\begin{gathered}
Z \equiv \frac{m_{B} U_{P}}{\hbar^{2} \sqrt{k_{L} k_{R}}}, \\
g \equiv \frac{m_{B} M_{\perp}}{\hbar^{2} \sqrt{k_{L} k_{R}}}, \\
g^{\prime} \equiv \frac{m_{B} M_{\|}}{\hbar^{2} \sqrt{k_{L} k_{R}}} .
\end{gathered}
$$

Note the appearance of the effective mass $m_{B}$ within the barrier region in Eq. (21). Although the barrier region is approximated to be infinitesimally small, in any realistic situation it will be sufficiently thick to define an effective mass.
Since we are interested in the Andreev bound states, our solution for $\Phi_{\nu}(z)$ must vanish in the limit $|z| \rightarrow \infty$. We hence adopt the ansatz

$$
\Phi_{\nu}(z)=e^{\sigma_{\nu} \kappa_{\nu} z}\left(\Phi_{\nu,+} e^{+i p_{\nu} z}+\Phi_{\nu,-} e^{-i p_{\nu^{z}}}\right),
$$

where

$$
\Phi_{\nu, \pm}=\left(u_{\nu, \pm}, v_{\nu, \pm}, w_{\nu, \pm}, x_{\nu, \pm}\right)^{T}
$$

are vectors describing right-moving $\Phi_{\nu,+}$ and left-moving $\Phi_{\nu,-}$ solutions, $\kappa_{\nu}$ and $p_{\nu}$ are real and positive, and we have $\sigma_{\nu}=+1(-1)$ for $\nu=L(R)$. The ansatz Eq. (22) corresponds to the quasiclassical approximation: the wave function is factored into a rapidly oscillating component $\left[\exp \left( \pm i p_{\nu} z\right)\right]$ and a slowly decaying envelope function $\left[\exp \left(\sigma_{\nu} \kappa_{\nu} z\right)\right]$.

In the bulk superconductors, we find after inserting Eq. (22) into Eq. (17) that the BdG equations simplify to a pair of $2 \times 2$ eigensystems: 


$$
\begin{gathered}
\left(\begin{array}{cc}
-\frac{\hbar^{2}}{2 m_{\nu}}\left(\sigma_{\nu} \kappa_{\nu} \pm i p_{\nu}\right)^{2}-\mu_{\nu} & i e^{-i\left(\theta_{\nu}-\phi_{\nu}\right)} \Delta_{\nu}\left(\sigma_{\nu} \kappa_{\nu} \pm i p_{\nu}\right) \\
i e^{i\left(\theta_{\nu}-\phi_{\nu}\right)} \Delta_{\nu}\left(\sigma_{\nu} \kappa_{\nu} \pm i p_{\nu}\right) & \frac{\hbar^{2}}{2 m_{\nu}}\left(\sigma_{\nu} \kappa_{\nu} \pm i p_{\nu}\right)^{2}+\mu_{\nu}
\end{array}\right) \\
\quad \times\left(\begin{array}{l}
u_{\nu, \pm} \\
v_{\nu, \pm}
\end{array}\right)=E\left(\begin{array}{l}
u_{\nu, \pm} \\
v_{\nu, \pm}
\end{array}\right), \\
\left(\begin{array}{ll}
-\frac{\hbar^{2}}{2 m_{\nu}}\left(\sigma_{\nu} \kappa_{\nu} \pm i p_{\nu}\right)^{2}-\mu_{\nu} & -i e^{i\left(\theta_{\nu}+\phi_{\nu}\right)} \Delta_{\nu}\left(\sigma_{\nu} \kappa_{\nu} \pm i p_{\nu}\right) \\
-i e^{-i\left(\theta_{\nu}+\phi_{\nu}\right)} \Delta_{\nu}\left(\sigma_{\nu} \kappa_{\nu} \pm i p_{\nu}\right) & \frac{\hbar^{2}}{2 m_{\nu}}\left(\sigma_{\nu} \kappa_{\nu} \pm i p_{\nu}\right)^{2}+\mu_{\nu}
\end{array}\right) \\
\quad \times\left(\begin{array}{l}
w_{\nu, \pm} \\
x_{\nu, \pm}
\end{array}\right)=E\left(\begin{array}{l}
w_{\nu, \pm} \\
x_{\nu, \pm}
\end{array}\right) .
\end{gathered}
$$

Both Eqs. (24) and (25) yield the same eigenvalue equations

$$
E^{2}=-\left(\sigma_{\nu} \kappa_{\nu} \pm i p_{\nu}\right)^{2} \Delta_{\nu}^{2}+\left(\frac{\hbar^{2}\left(\sigma_{\nu} \kappa_{\nu} \pm i p_{\nu}\right)^{2}}{2 m_{\nu}}+\mu_{\nu}\right)^{2} .
$$

The right-hand side of Eq. (26) must be real and positive. As the Andreev bound state wave functions are exponentially decaying in the bulk superconductors, we require that they be subgap solutions, i.e., have energy $|E|<k_{\nu} \Delta_{\nu}$. This is possible only if $\kappa_{\nu}$ and $p_{\nu}$ are nonzero; by setting the imaginary part of Eq. (26) to zero we obtain the relation

$$
p_{\nu}^{2}=k_{\nu}^{2}+\kappa_{\nu}^{2}-\frac{2 m_{v}^{2}}{\hbar^{4}} \Delta_{\nu}^{2}
$$

and therefore

$$
E^{2}=\left[\frac{\hbar^{2} k_{v}^{2}}{m_{\nu}}-\left(\frac{m_{\nu}}{\hbar^{2}} \Delta_{\nu}^{2}-\frac{\hbar^{2} \kappa_{v}^{2}}{m_{\nu}}\right)\right]\left(\frac{m_{\nu}}{\hbar^{2}} \Delta_{\nu}^{2}-\frac{\hbar^{2} \kappa_{v}^{2}}{m_{\nu}}\right) .
$$

This expression may be considerably simplified by observing that in physically realistic situations the maximum gap magnitude is much smaller than the Fermi energy, i.e., $k_{\nu} \Delta_{\nu}$ $\ll \mu_{\nu}=\hbar^{2} k_{\nu}^{2} / 2 m_{\nu}$. Furthermore, from the requirement that $E^{2}$ be positive we deduce that $\kappa_{\nu} \ll k_{\nu}$, hence allowing us to approximate Eq. (28) by

$$
E^{2}=k_{\nu}^{2} \Delta_{\nu}^{2}-\left(\frac{\hbar^{2} k_{\nu} \kappa_{\nu}}{m_{\nu}}\right)^{2} .
$$

Since we have $E^{2}<k_{\nu}^{2} \Delta_{\nu}^{2}$, this is a subgap solution, as we require. Furthermore, using the inequalities $\kappa_{\nu}, 2 m_{\nu} \Delta_{\nu} / \hbar^{2} \ll k_{\nu}$, we see from Eq. (27) that $p_{\nu} \approx k_{\nu}$; in this limit the solutions of Eqs. (24) and (25) become

$$
\begin{gathered}
v_{\nu \pm}=\frac{E \mp \sigma_{\nu} i \hbar^{2} k_{\nu} \kappa_{\nu} / m_{\nu}}{\mp e^{-i\left(\theta_{\nu}-\phi_{\nu}\right)} k_{\nu} \Delta_{\nu}} u_{\nu, \pm}, \\
x_{\nu, \pm}=\frac{E \mp \sigma_{\nu} i \hbar^{2} k_{\nu} \kappa_{\nu} / m_{\nu}}{ \pm e^{i\left(\theta_{\nu}+\phi_{\nu}\right)} k_{\nu} \Delta_{\nu}} w_{\nu, \pm} .
\end{gathered}
$$

Note that $\kappa_{\nu}$ is explicitly dependent upon $E$, and we obtain by solving Eq. (29)

$$
\kappa_{\nu}=\sqrt{k_{\nu}^{2} \Delta_{\nu}^{2}-E^{2}} / \hbar v_{F \nu},
$$

where $v_{F \nu}=\hbar k_{\nu} / m_{\nu}$ is the Fermi velocity on the $\nu$ side of the barrier.

We still need to find the Andreev bound state energies. This may be achieved by applying the boundary conditions (19) and (20) to the ansatz (22), and using (30) and (31). See Appendix B for more details and the explicit equation (B1) for the energies. An analytic solution of this equation is possible in the natural situation when the superconductors on either side of the gap are made from the same material, i.e., we have $k_{L}=k_{R} \equiv k_{F}, m_{L}=m_{R}$, and $k_{L} \Delta_{L}=k_{R} \Delta_{R} \equiv k_{F} \Delta_{0}$. We find four bound states $\pm E_{a, b}$, with positive branch

$$
E_{a(b)}=k_{F} \Delta_{0} \sqrt{D}|\sqrt{D A+B}+(-) \sqrt{D A-B}|,
$$

where

$$
\begin{aligned}
A= & \frac{1}{4}\left[\left(1+2 g^{2}+g^{\prime 2}+Z^{2}\right)+\left(1+g^{\prime 2}+Z^{2}\right) \cos \phi \cos \theta\right. \\
& \left.+g^{2} \cos (\theta-2 \alpha)(\cos \theta-\cos \phi)+2 Z g^{\prime} \sin \theta \sin \phi\right],
\end{aligned}
$$

$$
\begin{aligned}
& B=\frac{1}{2} \cos \frac{1}{2}(\theta-\phi) \cos \frac{1}{2}(\theta+\phi), \\
& D=\frac{1}{\sqrt{\left(1+g^{2}+g^{\prime 2}-Z^{2}\right)^{2}+4 Z^{2}}} .
\end{aligned}
$$

We consider Eq. (33) to be the central result of our analytic considerations.

The last remaining step is the calculation of the Josephson current $I_{J}$. This is given by ${ }^{14}$

$$
I_{J}=-\frac{e}{\hbar} \sum_{l=a, b} \frac{\partial E_{l}}{\partial \phi} \tanh \left(E_{l} / 2 k_{B} T\right),
$$

where $k_{B}$ is Boltzmann's constant. Since we are mostly interested in the low-temperature current, where the transport through the Andreev states dominates, by using Eq. (33) we may obtain an explicit expression for $I_{J}$ at $T=0$ :

$$
I_{J}=-\frac{e}{\hbar}\left(\frac{\partial E_{a}}{\partial \phi}+\frac{\partial E_{b}}{\partial \phi}\right)=-\frac{e k_{F} \Delta_{0}}{\hbar} \sqrt{\frac{D}{D A+|B|}} \frac{\partial(D A+|B|)}{\partial \phi},
$$

where, for later reference, we have explicitly

$$
\begin{aligned}
\frac{\partial(D A+|B|)}{\partial \phi}= & \frac{1}{4}\left\{D\left[g^{2} \cos (\theta-2 \alpha)-\left(1+g^{\prime 2}+Z^{2}\right) \cos \theta\right]\right. \\
& -\operatorname{sgn} B\} \sin \phi+\frac{1}{2} D Z g^{\prime} \sin \theta \cos \phi .
\end{aligned}
$$

Note that we henceforth work in units where $\hbar=1$.

\section{RESULTS}

In this section we study the TFT junction in the special case $k_{L}=k_{R} \equiv k_{F}, m_{L}=m_{R}$, and $k_{L} \Delta_{L}=k_{R} \Delta_{R} \equiv k_{F} \Delta_{0}$. We inves- 

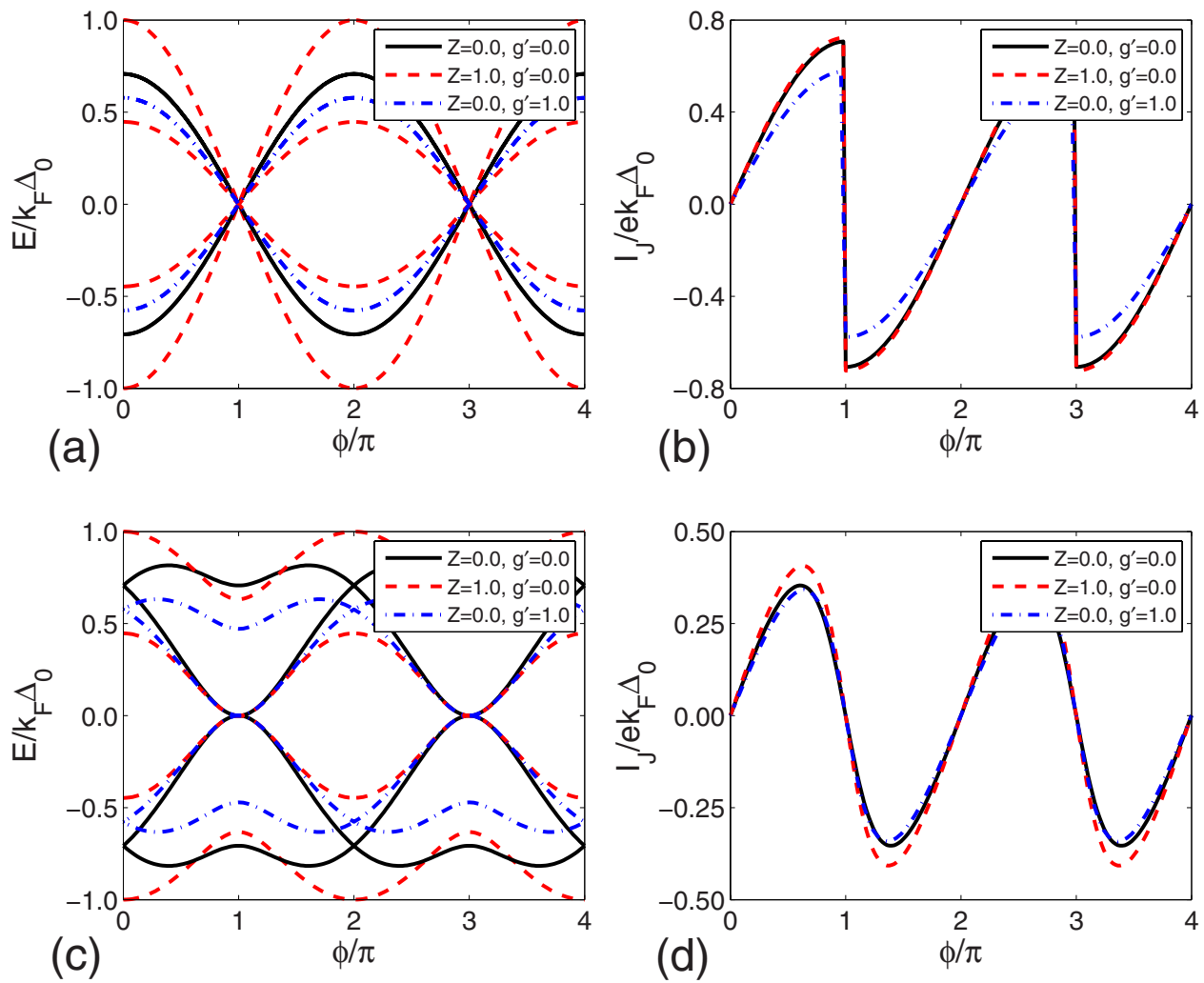

FIG. 2. (Color online) $\phi$ dependence of (a) the Andreev states and (b) the corresponding Josephson current for $g=1.0$, $\theta=0$, and $\alpha=\pi / 2 ; \phi$ dependence of (c) the Andreev states and (d) the corresponding Josephson current for $g=1.0, \theta=0$, and $\alpha=\pi / 4$.

tigate the dependence of the Andreev state energies [Eq. (33)] and the current through them [Eq. (37)] on the different junction parameters. In Sec. III A we discuss the Andreev state energies and current for the case of aligned $\boldsymbol{d}$ vectors, with a discussion of the temperature dependence of $I_{J}$ given in Sec. III A 1. The more general scenario with nonaligned $\boldsymbol{d}$ vectors is presented in Sec. III B. Lastly, in Sec. III C we consider the critical current as a function of $g, Z, \theta$, and $\alpha$ for a junction with $g^{\prime}=0$. Unless otherwise stated, all results are for $T=0$.

\section{A. Aligned $d$ vectors}

We first consider the case when the $\boldsymbol{d}$ vectors of the left and right superconductors are aligned, i.e., $\theta=0$. As was shown in Ref. 24, for a barrier with only $g \neq 0$, the $\phi$ dependence of the current depends crucially upon the alignment of the transverse component of the barrier magnetization with the $\boldsymbol{d}$ vectors. For $\boldsymbol{M} \perp \boldsymbol{d}_{L, R}$, i.e.,

$$
\alpha=\alpha_{c} \equiv(2 n+1) \pi / 2, \quad n \in \mathbb{Z},
$$

it was found that the Andreev states are degenerate and Eq. (33) simplifies to

$$
E_{a, b}=k_{F} \Delta_{0} \sqrt{D} \cos (\phi / 2),
$$

which is plotted as the black solid line in Fig. 2(a). This result is identical to the Andreev state energies obtained at a potential scattering barrier (i.e., $Z \neq 0$ only), ${ }^{21}$ as well as for a barrier with only a longitudinal moment $\left(g^{\prime} \neq 0\right.$ only) (see Fig. 4). Because the zero crossings at

$$
\phi=\phi_{Z C} \equiv(2 n+1) \pi, \quad n \in \mathbb{Z},
$$

occur with $\partial E_{a, b} / \partial \phi \neq 0$, we hence find discontinuous jumps in $I_{J}$ [shown in Fig. 2(b)]. The degeneracy of the Andreev states is lifted when there is a component of the magnetic moment parallel to the $\boldsymbol{d}$ vectors (i.e., $\alpha \neq \alpha_{c}$ ). This holds for all $\phi$ except for the level crossings located at

$$
\phi=\phi_{L C} \equiv 2 n \pi, \quad n \in \mathbb{Z},
$$

as shown in Fig. 2(c). At $\phi=\phi_{Z C}$ both the $a$ and $b$ states have a stationary point with respect to $\phi$, and the discontinuity in the current is therefore removed [Fig. 2(d)].

Including a finite potential barrier $Z$ when $g \neq 0$ has a very significant effect upon the $\phi$ dependence of the Andreev states, in particular it lifts the degeneracy of the states at $\alpha$ $=\alpha_{c}$ and removes the level crossings at $\phi=\phi_{L C}$ for $\alpha \neq \alpha_{c}$ [Figs. 2(a) and 2(c), respectively]. In the latter case, the $\phi$ dependence of $E_{a}$ is strongly modified by $Z$ : in Fig. 2(c) we see that not only does $E_{a}$ exhibit almost sinusoidal variation with $\phi$ at $Z=1$, but also the sign of $\partial E_{a} / \partial \phi$ is reversed relative to the $Z=0$ case in the vicinity of $\phi=\phi_{L C}$. This has interesting implications for the Josephson current, which displays a moderate enhancement above the $Z=0$ values for all $\phi$, as can be seen in Fig. 2(d). Because of the modification of $E_{a}$ near $\phi_{L C}$, the contribution to $I_{J}$ from the $a$ and $b$ states is of the same sign for all values of $\phi$. Furthermore, although 

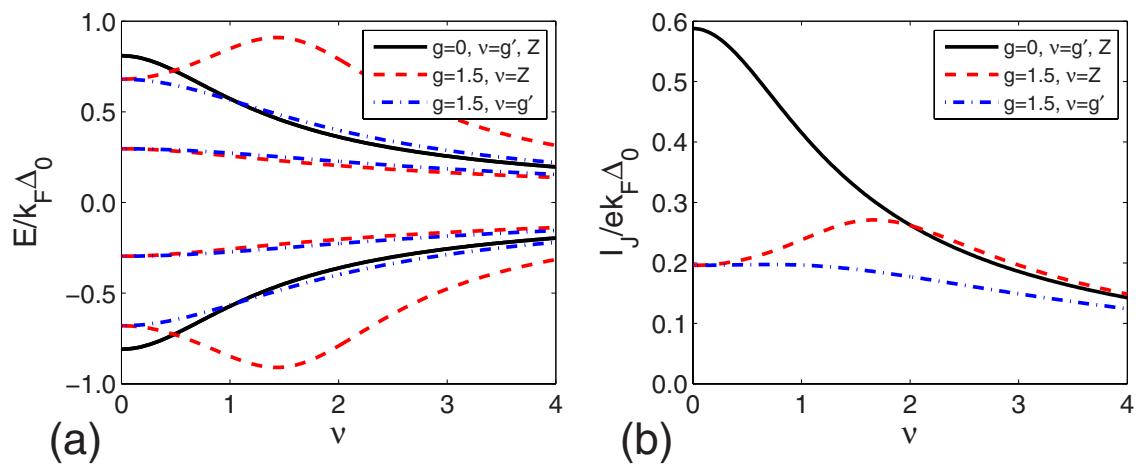

FIG. 3. (Color online) (a) Andreev state energies and (b) Josephson current as a function of $\nu$, where $\nu$ is either $Z$ or $g^{\prime}$. Note that $Z$ $=0$ if $\nu=g^{\prime}$ and vice versa. We assume $\theta=0, \phi=2 \pi / 5$, and $\alpha=\pi / 4$.

$\left|E_{b}\right|$ and hence also the current contributed by the $b$ state is reduced by a finite $Z$ [Fig. 3(a)], the increase in $\left|E_{a}\right|$ for $Z$ $<g$ can compensate, leading to the overall enhancement of $I_{J}$ at all $\phi$ values. At higher values of $Z$, the monotonic decrease in $\left|E_{a}\right|$ causes a reduction in $I_{J}$ and we therefore obtain the current maximum shown in Fig. 3(b). In contrast, for $\alpha$ $=\alpha_{c}$, the maximum in $I_{J}$ is very much reduced, as there is no change in the curvature of the Andreev states and so the maximum is due solely to the maximum in $\left|E_{a}\right|$ (not shown).

The change in the $\phi$ dependence of the Andreev states with the inclusion of a finite $g^{\prime}$ is much less dramatic than for a finite $Z$. The degeneracy of the Andreev states at $\alpha$ $=\alpha_{c}$ and the level crossings at $\alpha \neq \alpha_{c}$ both remain intact when $g^{\prime} \neq 0$, as seen in Figs. 2(a) and 2(c), respectively. At $\alpha=\alpha_{c}$ the only effect of $g^{\prime}$ is to narrow the allowed range of Andreev state energies, thus leading to a suppression of $I_{J}$ as seen in Fig. 2(b). For $\alpha \neq \alpha_{c}$ the reduction in the $\left|E_{a, b}\right|$ is not uniform, as evidenced in Fig. 2(c) by the greater decrease in the magnitude of $E_{a}$ at $\phi=\phi_{Z C}$ compared to that at $\phi=\phi_{L C}$. Although the modification of $E_{a}$ can lead to very weak increases in $I_{J}$ for $\phi$ close to $\phi_{Z C}$, only a monotonic decrease in the current with increasing $g^{\prime}$ is observed for other values of $\phi$ [e.g., see Fig. 3(b)].

We now consider the case $g=0$. As can be seen from the $\mathrm{BdG}$ equation (17), the absence of a transverse component of the barrier magnetization means that spin is a good quantum number. This has the interesting consequence that the effective barrier potentials can take different values in the two spin channels: for the spin- $\sigma$ electrons there is a combined charge and magnetic barrier of value $Z-\sigma g^{\prime}$ where $\sigma=+(-)$ for spin- $\uparrow(\downarrow)$. We may therefore label the Andreev state energies by $\sigma$, obtaining from Eq. (33) the expression

$$
E_{\sigma}=k_{F} \Delta_{0} \sqrt{D_{\sigma}} \cos (\phi / 2),
$$

where $D_{\sigma}=\left[1+\left(Z-\sigma g^{\prime}\right)^{2}\right]^{-1}$. We thus require that both potential and magnetic terms be present in the barrier to achieve a spin splitting of the Andreev states. This is shown in Fig. 4(a), where the $Z=0$ states (black lines) are split into the states $E_{\downarrow}$ (inner red broken lines) and $E_{\uparrow}$ (outer red broken lines). Due to the zero crossings of the Andreev states, we find discontinuous jumps in the current [Fig. 4(b)]; as can also be seen in Fig. 4(b), a finite $Z$ can give a slight enhancement of $I_{J}$ when $g^{\prime} \neq 0$. This is due to the maximum in $\left|E_{\uparrow}\right|$ which occurs when the potential and magnetic terms in the spin- $\uparrow$ sector cancel each other at $Z=g^{\prime}$. As the magnitude of the current flowing through the spin- $\uparrow$ states $\left(I_{J \uparrow}\right)$ is greater than the current flowing through the spin- $\downarrow$ states $\left(I_{J \downarrow}\right)$, there will be a Josephson spin current flowing through the junction, with $z$ component

$$
I_{J z}=-\frac{\hbar}{2 e}\left(I_{J \uparrow}-I_{J \downarrow}\right) .
$$

The occurrence of a Josephson spin supercurrent has been studied for tunneling between both unitary ${ }^{22}$ and nonunitary ${ }^{30,31}$ triplet superconductors, as well as singlet su-

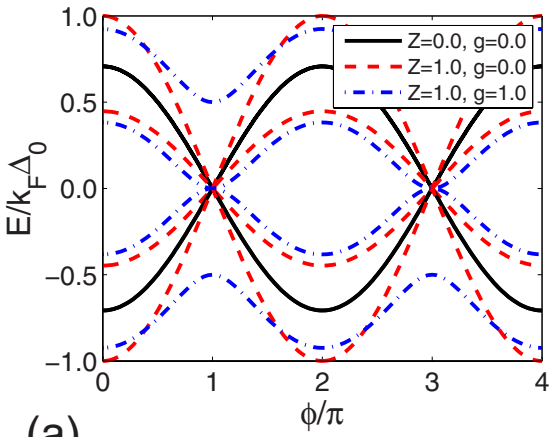

(a)

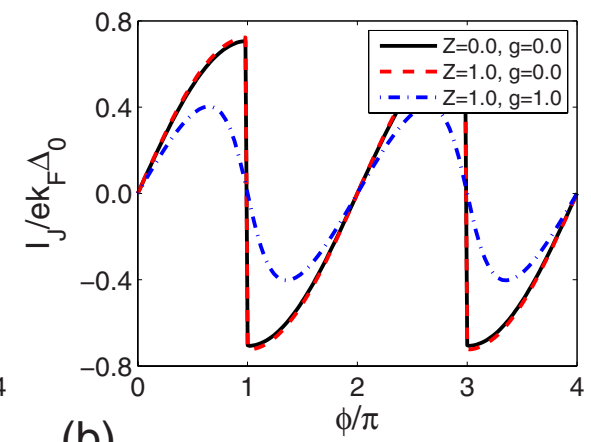

(b)

FIG. 4. (Color online) $\phi$ dependence of (a) the Andreev states and (b) the corresponding Josephson current for $g^{\prime}=1.0$ and $\theta=0$. $\alpha$ is undefined when $g=0$; for $g \neq 0$, we take $\alpha=\pi / 4$. 

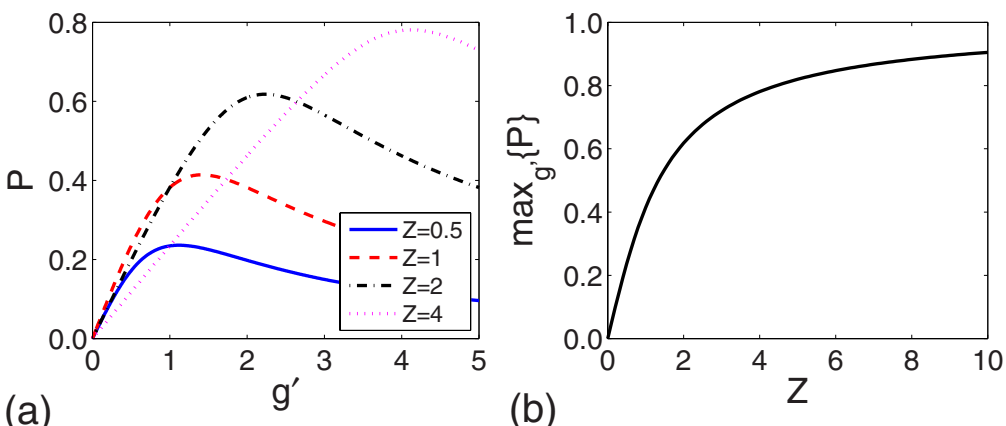

(b)

FIG. 5. (Color online) (a) Spin polarization $P$ of the Josephson current as a function of $g^{\prime}$ for various values of $Z$. We have $g=\alpha=\theta$ $=0$ for all lines. (b) Maximum spin polarization of the current with respect to $g^{\prime}$ as a function of $Z$. For given $Z$, the maximum in $P$ occurs at $g^{\prime}=g_{\max }^{\prime}=\sqrt{1+Z^{2}}$.

perconductors coexisting with helimagnetic order. ${ }^{34}$ A spin current can flow through a bulk unitary superconductor since it does not imply a change in the density of the two species of Cooper pairs. In the situation considered here, the current flowing through each spin sector is a constant multiple $\sqrt{D_{\sigma}} /\left(\sqrt{D_{\uparrow}}+\sqrt{D_{\downarrow}}\right)$ of the total current, and it is therefore possible to define a current polarization

$$
P=\frac{I_{J \uparrow}-I_{J \downarrow}}{I_{J \uparrow}+I_{J \downarrow}}=\frac{\sqrt{1+\left(Z+g^{\prime}\right)^{2}}-\sqrt{1+\left(Z-g^{\prime}\right)^{2}}}{\sqrt{1+\left(Z+g^{\prime}\right)^{2}}+\sqrt{1+\left(Z-g^{\prime}\right)^{2}}} .
$$

As a function of $g^{\prime}$, the polarization takes a maximum at $g_{\text {max }}^{\prime}=\sqrt{1+Z^{2}}$, shown in Fig. 5(a). This maximum value of the polarization asymptotically approaches 1 (i.e., fully spinpolarized current) as $Z \rightarrow \infty$ and hence also $g_{\max }^{\prime} \rightarrow \infty$ [Fig. $5(\mathrm{~b})$. In a realistic system, the spin current through the junction may be affected by spin-relaxation processes in the bulk superconductors, e.g., spin-orbit scattering. Including these effects requires a much more sophisticated treatment, however, and will therefore not be considered here.

When all barrier parameters are nonzero [the blue dotdashed line in Fig. 4(a)], the $\phi$ dependence of the Andreev states most closely resembles that of the $g, Z \neq 0, g^{\prime}=0$ case [Fig. 2(c)]. Referring to Fig. 4(a), we see that for $\alpha \neq \alpha_{c}$ not only are the $a$ and $b$ states nondegenerate at $\phi_{Z C}$, but they also have stationary points there, hence removing the discontinuities in $I_{J}$. At $\alpha=\alpha_{c}$, the states are degenerate at $\phi=\phi_{Z C}$ and show simple cosine dependence on $\phi$ as in Eq. (41), but with distinct amplitudes $D_{b(a)}=\left[1+\left(Z+(-) \sqrt{g^{2}+g^{\prime 2}}\right)^{2}\right]^{-1}$ (not shown).

As displayed in Fig. 6(a), the presence of the other barrier potentials substantially enhances the current for $g>1$. At smaller values of $g$, there is only a monotonic depression of the current with increasing $g^{\prime}$ and $Z$. We do not observe a maximum in $I_{J}$ as a function of $g$ for any choice of the other barrier potentials. In contrast, increasing $Z$ at fixed $g$ and $g^{\prime}$ [Fig. 6(b)] leads to a clear maximum at $Z \sim g+g^{\prime}$, with a greater peak current than at $g^{\prime}=0$. Defining the maximum current enhancement by the potential scattering $Z$ as

$$
\delta_{Z} I_{J}=\max _{Z}\left\{I_{J}\right\}-I_{J}(Z=0),
$$

where $\max _{Z}\left\{I_{J}\right\}$ is the maximum value of $I_{J}$ with respect to $Z$ with $\phi$ and the other barrier potentials fixed, we therefore see in Fig. 6(b) that $\delta_{Z} I_{J}$ increases with increasing $g^{\prime}$. When $Z$ $=0$, the current as a function of $g^{\prime}$ shows only a weak maximum at $g>1$ which is most pronounced at fixed $\phi \sim \phi_{Z C}$ (not shown); in the presence of a potential scattering term $Z>1$, however, $\left|I_{J}\right|$ develops a clear maximum at $g^{\prime} \sim Z$ for all $\phi$ as shown in Fig. 6(c). The current enhancement when all three barrier parameters are nonzero is most pronounced when $g^{\prime} \sim Z \gg g, 1$; in this limit we approach the case $g=0$, when the maximum is due to the cancellation of the magnetic and potential scattering terms in the spin- $\uparrow$ channel, and there is consequently very little $g$ dependence of the current [Fig. 6(a)]. Thus, the maxima in Figs. 6(b) and 6(c) occur in spite of and not because of $g \neq 0$.
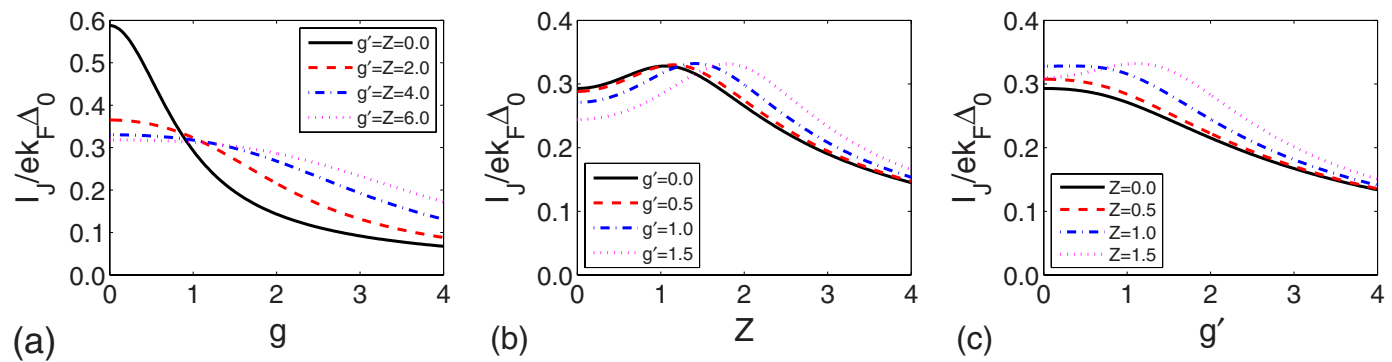

FIG. 6. (Color online) Comparison of the dependence of the current on the barrier potentials for $\theta=0, \alpha=\pi / 4$, and $\phi=2 \pi / 5$. Current as a function of (a) $g$ for different values of $g^{\prime}=Z$; (b) $Z$ for different values of $g^{\prime}$ and fixed $g=1$; (c) $g^{\prime}$ for different values of $Z$ and fixed $g=1$. 


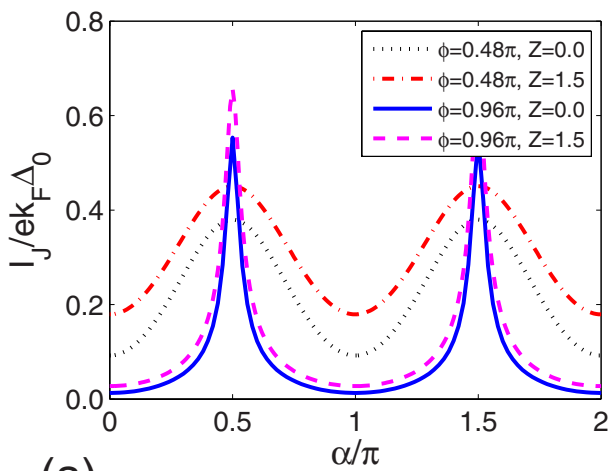

(a)

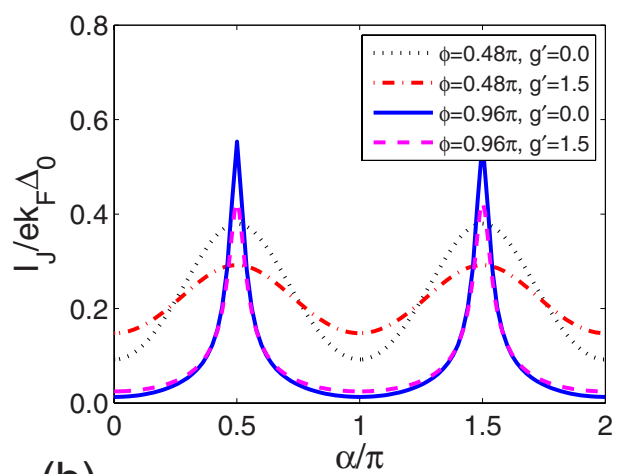

(b)

FIG. 7. (Color online) Effect of (a) $Z \neq 0$ and (b) $g^{\prime} \neq 0$ on the $\alpha$ dependence of the current for $g=1, \theta=0$, and various values of $\phi$.

It is interesting to compare the dependence of $I_{J}$ on $g^{\prime}$ and $Z$ for $g=0$ to the results for a singlet superconductorferromagnet-singlet superconductor (SFS) junction: ${ }^{29}$ although a maximum in the current through the SFS junction is found as a function of $Z$ at constant $g^{\prime}$, the value of $I_{J}$ at this maximum $\left(Z \sim g^{\prime}\right)$ vanishes as $Z, g^{\prime} \rightarrow \infty$. In the case here, in contrast, the current at the maximum approaches a finite value in this limit. The difference can be understood as arising from the fact that, in the singlet case, the superconducting correlations are perturbed no matter in which spin channel the scattering occurs. Even if scattering occurs only in one of the spin channels (as is the case for $Z=g^{\prime}$ ), an effective scattering is induced in the other spin channel through the anomalous superconducting correlations. As a result, for $Z=g^{\prime}$ and $Z, g^{\prime} \rightarrow \infty$, the tunneling of electrons and hence the Josephson current are completely suppressed. In contrast, in the triplet case for $\boldsymbol{d}_{L, R} \perp \boldsymbol{M}$ and $Z=g^{\prime}$, only the superconducting correlations in the spin- $\downarrow$ channel (where the effective barrier potential is $\left.Z+g^{\prime}\right)$ are suppressed, while those in the spin- $\uparrow$ channel are not (here the effective barrier potential vanishes). As a result, the Josephson current remains finite in the limit $Z, g^{\prime} \rightarrow \infty$ and is solely carried by the spin- $\uparrow$ Andreev state.

The current displays a periodic modulation as the magnetic moment is rotated about the $z$ axis as shown in Fig. 7. The amplitude of the modulation increases as $\phi$ approaches $\phi_{Z C}$, with $I_{J}$ becoming sharply peaked at $\alpha=\alpha_{c}$ for $\phi \sim \phi_{Z C}$. In the limit $\phi \rightarrow \phi_{Z C}^{ \pm}=\phi_{Z C} \pm 0^{+}$, the current vanishes unless $\boldsymbol{M} \perp \boldsymbol{d}_{L, R}$. This behavior can be understood as follows. For all $\alpha \neq \alpha_{c}, E_{a, b}$ has a stationary point at $\phi=\phi_{Z C}$ [see Fig. 2(c)], and so $I_{J}$ vanishes as $\phi \rightarrow \phi_{Z C}$; for $\alpha=\alpha_{c}$, however, there is no stationary point at $\phi=\phi_{Z C}$ and so $\lim _{\phi \rightarrow \phi_{Z C}^{ \pm}} \partial E_{a, b} / \partial \phi \neq 0$, which thus gives a finite Josephson current. Close to $\phi$ $=\phi_{Z C}$, therefore, the current shows a "switch"-like dependence upon the orientation of $\boldsymbol{M}$ in the $x-y$ plane. That is, small variations in $\alpha$ can lead to large changes in the magnitude of $I_{J}$, switching the junction from the on state $\left(I_{J}\right.$ $\neq 0)$ to an off state $\left(I_{J} \approx 0\right)$. This Josephson current switch survives in the presence of a finite $Z$ or $g^{\prime}$, although there are significant differences between the two cases. For $g^{\prime} \neq 0$, we observe at $\alpha=\alpha_{c}$ a reduction in the magnitude of both $I_{J}$ and the amplitude of the current oscillations. For $Z \neq 0$, in contrast, there is an increase of the Josephson current at all val- ues of $\alpha$. The enhancement is almost $\alpha$ independent for $\phi$ $\sim \phi_{L C}$, whereas for $\phi \sim \phi_{Z C}$ the enhancement is concentrated at the $\alpha=\alpha_{c}$ peak, strengthening the switch effect.

\section{Temperature dependence}

In Ref. 24 it was predicted that for a superconducting gap with BCS temperature dependence $\Delta_{0}(T)$, the Josephson current through the TFT junction can reverse sign as the temperature $T$ is raised. This unconventional temperature dependence of $I_{J}$ is shown here in Fig. 8. The reversal of $I_{J}$ is a consequence of the splitting of the Andreev states by the transverse magnetic scattering terms in the barrier, which gives each state two distinct branches at $\pm\left|E_{a}\right|( \pm a)$ and $\pm\left|E_{b}\right|( \pm b)$. At $T=0$, only the $-a$ and $-b$ branches are occupied. Since $\left|\partial E_{a} / \partial \phi\right|<\left|\partial E_{b} / \partial \phi\right|$, the $-b$ branch makes the dominant contribution to the current and hence determines the direction of current flow. At any finite temperature the $+a$ and $+b$ branches have a nonzero population, with the occupation of the $+b$ branch always larger than that of the $+a$ branch since $\left|E_{a}\right|>\left|E_{b}\right|$. As the derivatives of the $+a$ and $+b$ branches are equal and opposite to those of the $-a$ and $-b$ branches, respectively, this causes a reduction of the contributions to the current from both the $a$ and $b$ states. Because we have $\left|E_{a}\right|>\left|E_{b}\right|$, however, the change in occupation (and hence also the reduction in current) is greatest for the $\pm b$ branches. If this reduction is sufficiently large the contribution of the $a$ state may eventually dominate the current. If the sign of $\partial E_{a} / \partial \phi$ is opposite to $\partial E_{b} / \partial \phi$, the direction of current flow then reverses. Since the $a$ and $b$ states are degenerate in the limits $g \rightarrow 0$ or $\alpha \rightarrow \alpha_{c}$, it is thus not surprising that, for example, when $\phi=\pi / 2$ and $\theta=g^{\prime}=Z=0$ the sign change of $I_{J}$ (i) occurs only for sufficiently large $g \gtrsim 1$ when $\alpha=0$ [Fig. 8(a)], and (ii) is entirely absent for $\pi / 4 \leqslant \alpha$ $\leqslant 3 \pi / 4$ [Fig. 8(b)].

As the $\phi$ dependence of the Andreev states is crucial for the temperature-dependent reversal of $I_{J}$, it is of interest to consider the robustness of this effect in the presence of nonzero $g^{\prime}$ or Z. As shown in Fig. 8(c), both these terms suppress the reversal of $I_{J}$, raising it to higher temperatures and reducing the maximum magnitude of the reversed current. The reason for this is due to the change in the $\phi$ dependence of the Andreev states, as illustrated in Fig. 8(d). For both $g^{\prime} \neq 0$ and $Z \neq 0$ the value of $\partial E_{b} / \partial \phi$ at $\phi=\pi / 2$ changes 

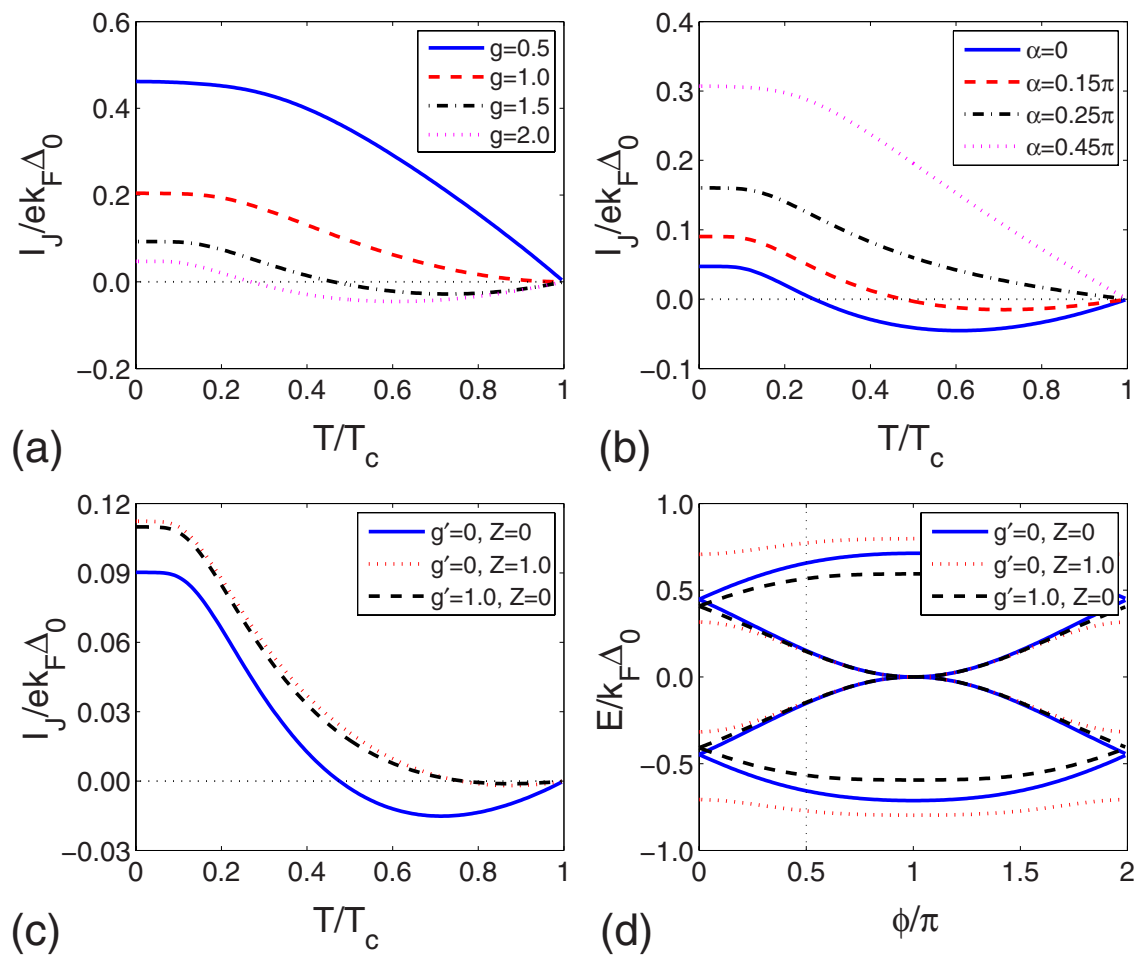

FIG. 8. (Color online) (a) Temperature dependence of the current for various values of $g$, with $\alpha=0, \theta=0, \phi=\pi / 2$, and $Z=g^{\prime}=0$. (b) Temperature dependence of the current for various values of $\alpha . g=2.0$ and other parameters are as in (a). (c) Effect of nonzero $g^{\prime}$ or $Z$ on temperature dependence of current at $\alpha=0.15 \pi$ and other parameters as for (b). (d) Andreev states as a function of $\phi$ for the parameters in (c). The dotted vertical line corresponds to the situation in (c).

very little from the $g^{\prime}=Z=0$ case; in contrast, the value of $\partial E_{a} / \partial \phi$ is considerably reduced. That is, the $a$ state has a proportionately lower contribution to the current. To achieve a sign reversal in $I_{J}$, therefore, a much higher temperature is required to sufficiently deplete the $-b$ branch. More generally, the tendency of a finite $Z$ or $g^{\prime}$ to enhance the $T=0$ current will suppress the sign-reversal effect: for example, when $g=2, \alpha=0.15 \pi$, and $\theta=g^{\prime}=0$, for $Z \gtrsim 1.5$ we find that the signs of $\partial E_{a} / \partial \phi$ and $\partial E_{b} / \partial \phi$ are the same at $\phi=\pi / 2$, hence making a temperature-dependent reversal of $I_{J}$ impossible (not shown).

The two key requirements for a sign change in $I_{J}$ with increasing temperature are that the Andreev states be nondegenerate and that the derivative with respect to $\phi$ of the two distinct states have opposite signs. As such, when $\theta=0$ this effect occurs only when the magnetic moment of the barrier has a finite transverse component. Because of the very general condition for its appearance, it is nevertheless not unique to the TFT junction considered here: a reversal of $I_{J}$ with increasing $T$ has also been predicted for a junction constructed by placing a magnetic barrier between two $s$-wave superconductors. ${ }^{29}$

\section{B. Nonaligned $d$ vectors}

We now consider the case when the $\boldsymbol{d}$ vectors of the left and right superconductors are not aligned, i.e., $\theta \neq 0$. This creates an interesting situation, as from Eqs. (24) and (25) we see that the effective phase difference between the left and right superconductors is spin dependent and given by $\phi$ $-\sigma \theta .^{21}$ We therefore first consider the case when $g=0$, where the Andreev states are spin polarized. The expression Eq. (44) for $E_{\sigma}$ thus becomes

$$
E_{\sigma}=k_{F} \Delta_{0} \sqrt{D_{\sigma}} \cos [(\phi-\sigma \theta) / 2],
$$

with $D_{\sigma}$ as in Eq. (44). We see that the effect of $\theta \neq 0$ is to shift the Andreev energies of each spin sector by $2 \theta$ relative to one another [black solid lines in Fig. 9(a)]. This produces two sets of zero crossings, at

$$
\phi_{n, \pm}=(2 n-1) \pi \pm \theta, \quad n \in \mathbb{Z},
$$

which are both evidenced in the current by jump discontinuities [see Fig. 9(d)]. Including now also a finite $Z$, the spin- $\uparrow$ and spin- $\downarrow$ Andreev states have different amplitudes [red broken line in Figs. 9(a) and 9(c)].

As in the $\theta=0$ case, there is a spin current through the junction. Here, however, it is possible that the spin current flows even when the charge current is vanishing, and so the concept of the polarization is not valid. For the states shown in Fig. 9(a), we plot the corresponding spin current $I_{J z}$ in Fig. 9(b). Comparing the spin current with the charge current shown in Fig. 9(d), we see that for $g^{\prime}=1, Z=g=0$, a spin current flows at $\phi=n \pi$ even when $I_{J}$ is vanishing. We find discontinuous jumps in the spin current occur at the zero crossings of the Andreev states, as the sign of the Josephson current through the spin- $\sigma$ states reverses as $\phi$ is increased past $\phi_{n, \sigma}=(2 n-1) \pi+\sigma \theta$. In the case $Z=0$, the sign of the 

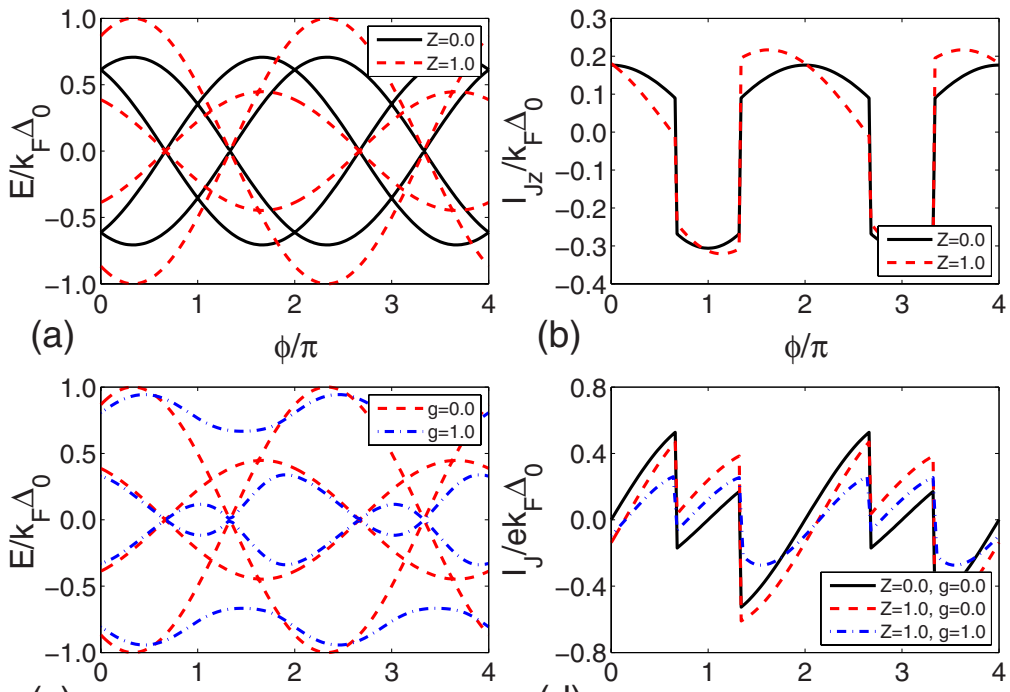

(c) $\phi / \pi$
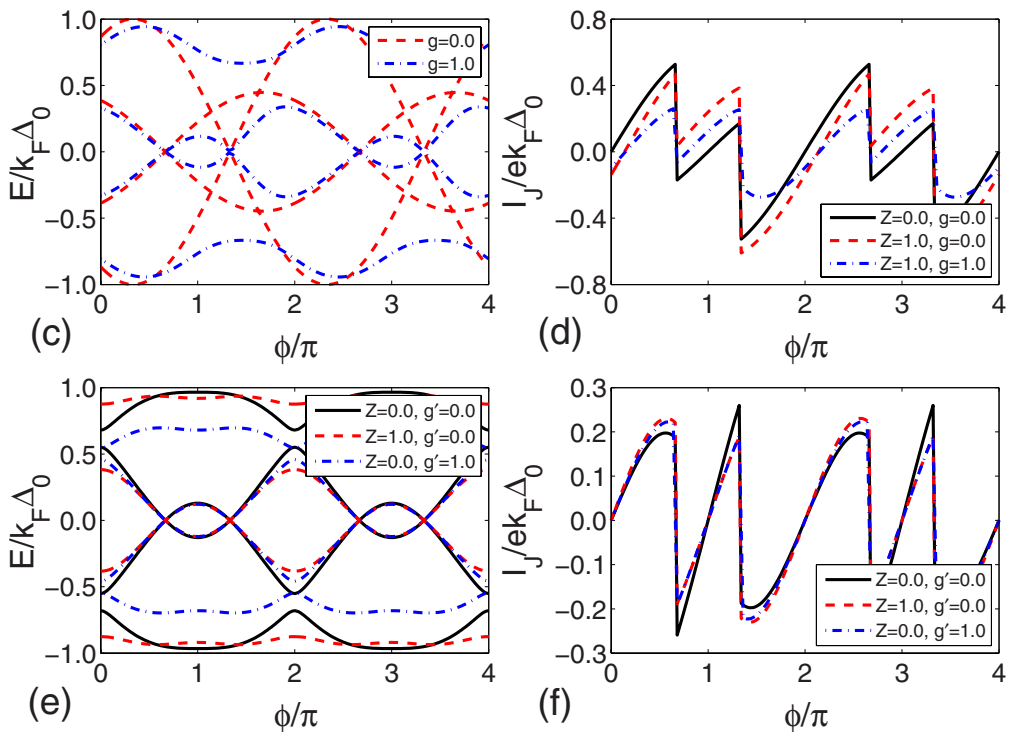

FIG. 9. (Color online) Comparison of the $\phi$ dependence of (a) the Andreev states and (b) the corresponding Josephson spin current both with and without a finite $Z$ for $g^{\prime}=1, g=0$, and $\theta=\pi / 3$. In (c) we show the effect of a finite $g$ on the $g^{\prime}=Z=1$ Andreev states. We take $\alpha=\pi / 4$ and $\theta=\pi / 3$. (d) The Josephson current flowing through the Andreev states shown in (a) and (c). $\phi$ dependence of (e) the Andreev states and (f) the corresponding Josephson current for $g=1.0, \theta=\pi / 3, \alpha=\pi / 4$, and at most one other barrier term nonzero.

spin current changes across the discontinuity; for $Z=1$ the spin current is negative on both sides of the jump discontinuity at $\phi_{n,+}$. The discontinuous sign changes in $I_{J z}$ as a function of $\phi$ may be thought of as a spin switch effect, in analogy to the current switch effect discussed below. An important difference from the case when $\theta=0$ is that we do not require any potential scattering at the barrier for the appearance of the spin current. Rather, as pointed out in Ref. 22, the misalignment of the $\boldsymbol{d}$ vectors causes a gradient of the spinspace order parameter, which produces a spin supercurrent in analogy to superfluid ${ }^{3} \mathrm{He} \cdot{ }^{35-38}$ It is therefore not necessary that the barrier be magnetically active: indeed, as can be seen in Eq. (48), even for a transparent barrier $g=g^{\prime}=Z=0$, the spin degeneracy of the Andreev states is lifted by the misalignment of the $\boldsymbol{d}$ vectors. As in the case when only $g^{\prime} \neq 0$, a spin current then flows at $\phi=0$.

Because of the nonalignment of the $\boldsymbol{d}$ vectors in the two superconductors, we see that if $Z, g^{\prime} \neq 0$ a current flows even when $\phi=0$, as clearly evidenced by Eqs. (38) and (39). Although it is still present when the barrier has a transverse magnetization, this result can be most easily understood when $g=0$ : in the spin- $\uparrow$ sector there is an effective phase difference of $-\theta$ and an effective barrier of $Z-g^{\prime}$; in the spin- $\downarrow$ sector there is an effective phase difference of $\theta$ and a barrier $Z+g^{\prime}$. Since the effective phases are of opposite signs, the Josephson current also flows in opposite directions in each spin sector. Assuming $Z, g^{\prime}>0$, we see that, as the effective barrier in the spin- $\downarrow$ sector is larger than in the spin- $\uparrow$ sector, the magnitude of the Josephson current through the former is smaller than that through the latter and hence there is a net current flow. This explanation is not readily applicable to the $g \neq 0$ case, as then the $z$ component of spin is no longer a good quantum number, but we nevertheless speculate that the $a$ and $b$ states retain some of the character of the $g=0$ states at finite $g$. As $g$ is increased, the mixing of the two spin sectors also increases and the current at $\phi=0$ is suppressed. A charge current at $\phi=0$ was predicted for a junction between a $p$-wave and an $s$-wave superconductor with spin-orbit coupling in the tunneling barrier, ${ }^{20}$ although our proposal involves a very different junction geometry, for the charge current to flow it similarly requires the breaking of time-reversal symmetry by the spin-dependent effective phases and barriers.

Another remarkable aspect of the results for $\theta \neq n \pi$ and $Z$, $g^{\prime} \neq 0$ is that the Andreev states are no longer symmetric about $\phi=n \pi$. As shown in Fig. 9(d), this has the striking result of removing the antisymmetry of $I_{J}$ about $\phi=n \pi$, which is present in all other cases. This asymmetry is also found in the presence of a finite $g$ (i.e., all barrier parameters nonzero). Despite significant changes in the $\phi$ dependence of the Andreev states in this case [blue dot-dashed line in Fig. $9(\mathrm{c})$ ], the zero crossings at $\phi_{n, \pm}$ remain: as is demonstrated 

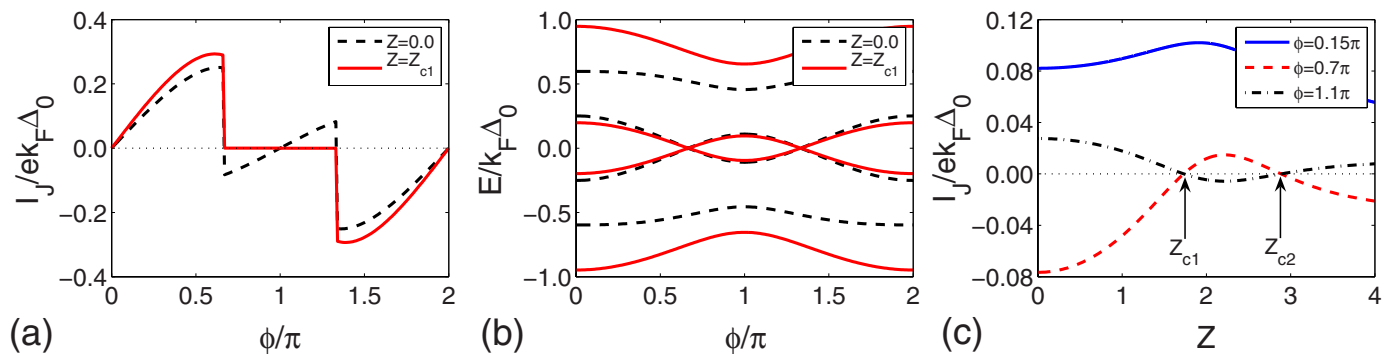

FIG. 10. (Color online) Comparison of (a) $I_{J}$ and (b) the Andreev states for $Z=0$ and $Z=Z_{c 1} \equiv 1.732051 \ldots$, with fixed $\theta=\pi / 3, g=2.0$, $g^{\prime}=0$, and $\alpha=\pi / 2$. The dotted line in (a) is a guide to the eye. The current at fixed $\phi$ is plotted as a function of $Z$ in (c) for the same parameter values as before. Note the sign change at the two critical values $Z_{c 1}$ and $Z_{c 2} \equiv 2.88675 \ldots$.

by Eq. (B3), the condition for zero-energy states is independent of the barrier.

We now turn to the case when $g \neq 0$, and at most one other barrier parameter is nonzero. Comparing Fig. 9(e) with Fig. 2(c), we see that, in addition to producing zero crossings at $\phi_{n, \pm}$, a finite $\theta$ also removes the level crossings at $\phi$ $=\phi_{L C}$ when $\alpha \neq \alpha_{c}$; for $\alpha=\alpha_{c}$ it also removes the level degeneracy (not shown). The effect of $Z \neq 0$ or $g^{\prime} \neq 0$ is qualitatively similar to that for $\theta=0$ : for both we observe a change in the sign of the curvature of $E_{a}$ around $\phi=\phi_{Z C}$, and for $Z \neq 0$ there is also a widening of the gap at $\phi=\phi_{L C}$, while for the case $g^{\prime} \neq 0$ there is a substantial narrowing of the range of allowed energies. Because the zero crossings of the Andreev states are controlled only by the relative orientation of the $\boldsymbol{d}$ vectors, jump discontinuities in $I_{J}$ are always present when $\theta \neq n \pi$ [see Figs. 9(d) and 9(f)]. As was discussed in Sec. III A 1 , for $\theta=0$, when the $a$ and $b$ states are nondegenerate, the $b$ state in general gives a greater contribution to $I_{J}$ than the $a$ state; numerical investigations show that this is also the case for $\theta \neq 0$. Because the contribution to the current from the $b$ states reverses sign for $\phi_{n,-}<\phi<\phi_{n,+}$, the jump discontinuities in Fig. 9(f) are therefore accompanied by a reversal of the sign of $I_{J}$. Furthermore, we note that the dependence of $I_{J}$ on $Z$ and $g^{\prime}$ for $\phi_{n,-}<\phi<\phi_{n,+}$ is very different from that for $\phi_{n-1,+}<\phi<\phi_{n,-}$ : for the latter we see an enhancement of the current, whereas $\left|I_{J}\right|$ is reduced in the former case. This is due to the changes in the $\phi$ dependence of the Andreev states, which tend to enhance the contribution to $I_{J}$ from the $a$ state in the region $\phi_{n,-}<\phi<\phi_{n,+}$, while leaving the contribution from the $b$ state mostly unaffected. As the contribution from the $a$ and $b$ states is of opposite sign for these values of $\phi$, this leads to an overall decrease in $\left|I_{J}\right|$. The current increase for $\phi_{n-1,+}<\phi<\phi_{n,-}$ is due to the subtle effects discussed in Sec. III A.

A remarkable example of the competing contributions from the $a$ and $b$ states is found in Fig. 10: for given $\theta$, it is sometimes possible to select barrier parameters such that the current from the $a$ and $b$ states completely cancel each other for a finite range of $\phi$. Analysis of Eq. (39) reveals that this occurs only for $g, Z \neq 0$ and $g^{\prime}=0$, when the condition

$$
D\left[g^{2} \cos (\theta-2 \alpha)-\left(1+Z^{2}\right) \cos \theta\right]-\operatorname{sgn} B=0
$$

is satisfied. As presented in Fig. 10(a) for $\theta=\pi / 3, g=2.0$, and $\alpha=\pi / 2$, a barrier potential of approximately $Z=Z_{c 1}$ $\equiv 1.732051 \ldots$ leads to a cancellation of the current for $\phi_{n,-}<\phi<\phi_{n,+}$. The current cancellation reflects a special relationship between the $a$ and $b$ states at $Z_{c 1}$ : for this choice of barrier potentials the moduli of the energies of the two states are equal up to a $\phi$-independent constant $C$, i.e.,

$$
E_{a}(\phi)= \begin{cases}E_{b}(\phi)+C, & \phi_{n-1,+}<\phi<\phi_{n,-}, \\ -E_{b}(\phi)+C, & \phi_{n,-}<\phi<\phi_{n,+} .\end{cases}
$$

Note from the definition Eq. (33) that $E_{a}(\phi)$ is always positive. When the contribution to the current from the $b$ state reverses sign for $\phi_{n,-}<\phi<\phi_{n,+}$, it exactly cancels the contribution to the current due to the $a$ state. On further increase of $Z$ above $Z_{c 1}$, the contribution to the current from the $a$ state dominates that from the $b$ state and hence the sign of the current for $\phi_{n,-}<\phi<\phi_{n,+}$ is reversed compared to the $Z=0$ case, as shown in Fig. 10(c). Although the current still displays discontinuities at $\phi_{n, \pm}$, it does not change sign across the intervals $\left(\phi_{n, \pm}-0^{+}, \phi_{n, \pm}+0^{+}\right)$. As $Z$ is increased a second zero-current state is found at $Z=Z_{c 2} \equiv 2.88675 \ldots$, above which the $b$ state again has the dominant contribution to $I_{J}$ and the sign of the current for $\phi_{n,-}<\phi<\phi_{n,+}$ is the same as at $Z=0$. We note that, since $\lim _{Z \rightarrow \infty} I_{J}=0$, the magnitude of the current for $\phi_{n,-}<\phi<\phi_{n,+}$ will take a maximum at some $Z>Z_{c 2}$, beyond which it asymptotically decreases (not shown).

\section{Rotating the d vectors: Equilibrium limit}

The jump discontinuity in $I_{J}$ observed in Fig. 9 can be used to construct another Josephson current switch. For fixed phase difference between the left and right superconductors, rotating $\boldsymbol{d}_{R}$ will cause a zero crossing of the $a$ states at

$$
\theta_{n, \pm}=(2 n+1) \pi \pm \phi, \quad n \in \mathbb{Z},
$$

and hence a jump discontinuity in the current as presented in Fig. 11. This clearly forms the basis of a switch effect, as for $\theta$ close to $\theta_{n, \pm}$, small changes between the relative alignment of the $\boldsymbol{d}$ vectors can switch the junction between different current states with opposite direction of $I_{J}$. The robustness of this switch effect, however, depends upon the ratio of the period of the rotation $T_{\theta}$ to the relaxation time $\tau$. In this section we consider the equilibrium limit where $T_{\theta} \gg \tau$ and so the system is always in thermal equilibrium; the opposite limit where $T_{\theta} \ll \tau$ is considered in the following section. In the equilibrium limit, the occupation of the Andreev levels is 


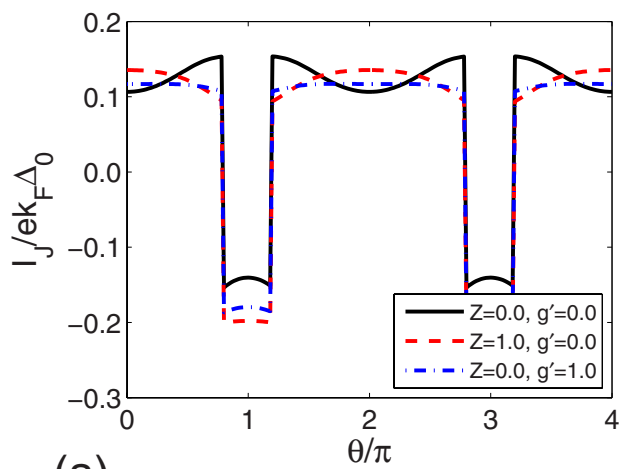

(a)

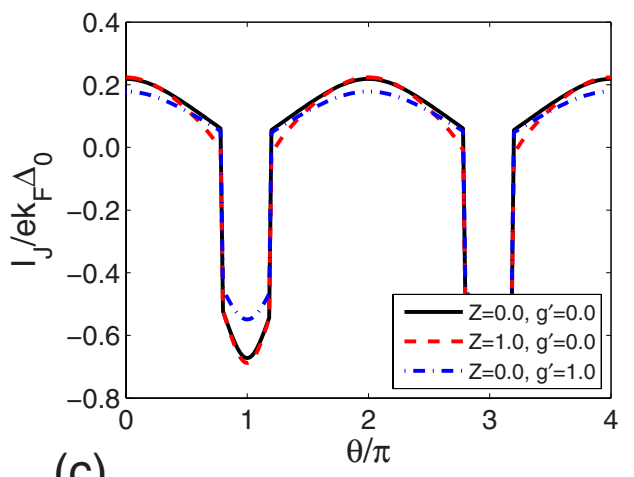

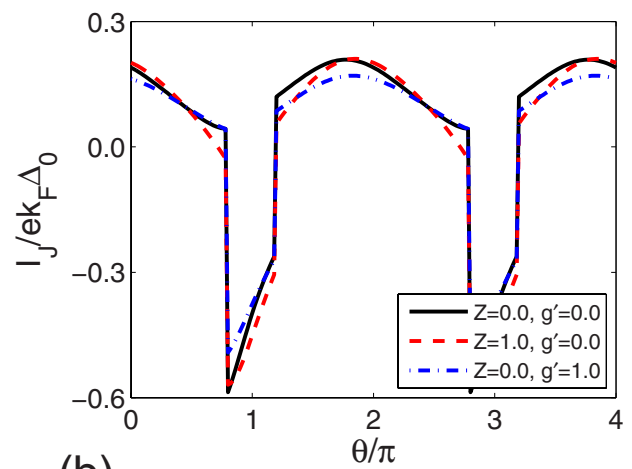

(b)

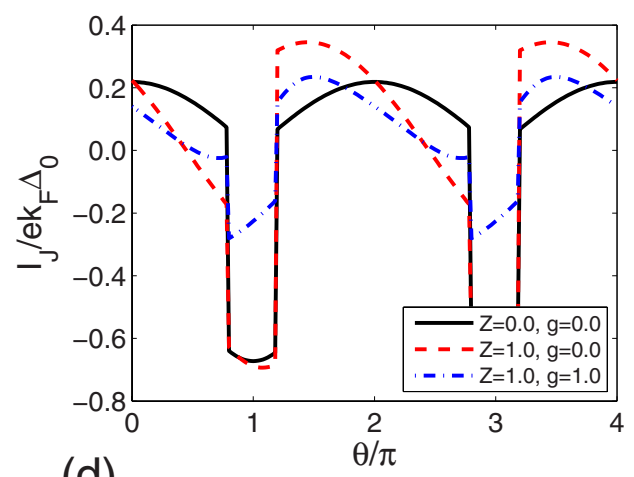

(d)

FIG. 11. (Color online) Current as a function of $\theta$ for various barrier parameter combinations with $g=1.0$ at $\phi=\pi / 5$ and $\alpha=0$ (a); (b) $\pi / 3$; (c) $\pi / 2$. (d) Current as a function of $\theta$ for various barrier parameter combinations with $g^{\prime}=1.0$ at $\phi=\pi / 5$ and $\alpha=0$.

given by the Fermi distribution throughout the period of the rotation.

In Ref. 24 it was noted that the $\theta$ dependence of the two current states can be significantly altered by changing the value of $\alpha$ [presented here in Figs. 11(a)-11(c)]. In particular, note that only at $\alpha=0$ and $\alpha=\alpha_{c}$ is the current symmetric with respect to $\theta$ about $\theta=n \pi$; for $\alpha$ intermediate between these values, the current is strongly skewed, varying almost linearly with $\theta$ in Fig. 11(b). The $\theta$ dependence of the current can also be modified by a finite $Z$ or $g^{\prime}$, as seen for example in Fig. 11(a) where we observe that the curvature with respect to $\theta$ of the "positive" and "negative" current states can reverse sign as $Z$ or $g^{\prime}$ is increased. As $\alpha$ is increased toward $\alpha_{c}$, however, the changes produced by nonzero $Z$ or $g^{\prime}$ become much less pronounced [e.g., Fig. 11(c)]. As shown in
Fig. 11(d), more radical alterations in the $\theta$ dependence of $I_{J}$ can be obtained when $g^{\prime}$ and $Z$ are both finite.

Close inspection of Fig. 11(b) for $Z=1$ shows that the current changes sign at $\theta \approx 0.7 \pi$, slightly before the jump discontinuity at $\theta=0.8 \pi$; also, when $Z=1$, a sign change is observed in Fig. 11(d) at $\theta \approx 0.5 \pi$ both with and without a finite $g$. These results indicate that the switch effect can be spoiled by sufficiently large $Z$ : although a jump discontinuity between the two different current states still occurs at $\theta_{n,-}$, the current does not reverse sign as we move from $\theta_{n,-}-0^{+}$to $\theta_{n,-}+0^{+}$. The similar behaviors in Figs. 11(b) and 11(d) nevertheless arise from qualitatively different $\phi$ dependencies of $I_{J}$, which can be seen in Fig. 12. We first consider the current corresponding to the $Z=1$ case in Fig. 11(b). Since $g^{\prime}=0, I_{J}$ is antisymmetric about $\phi=n \pi$ : as such, the continuous rever-
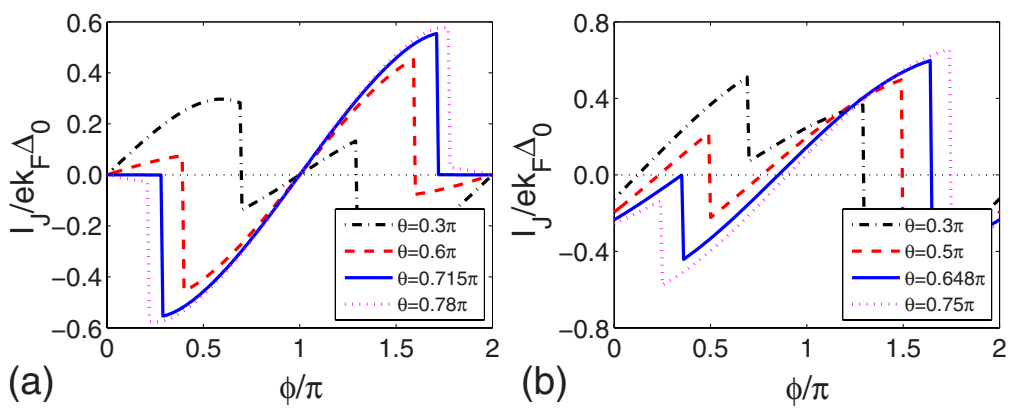

FIG. 12. (Color online) Current as a function of $\phi$ for $Z=1, \alpha=\pi / 3$ for various values of $\theta$, and for (a) $g=1.0, g^{\prime}=0, \alpha=\pi / 3$; (b) $g$ $=0.0, g^{\prime}=1.0$. 

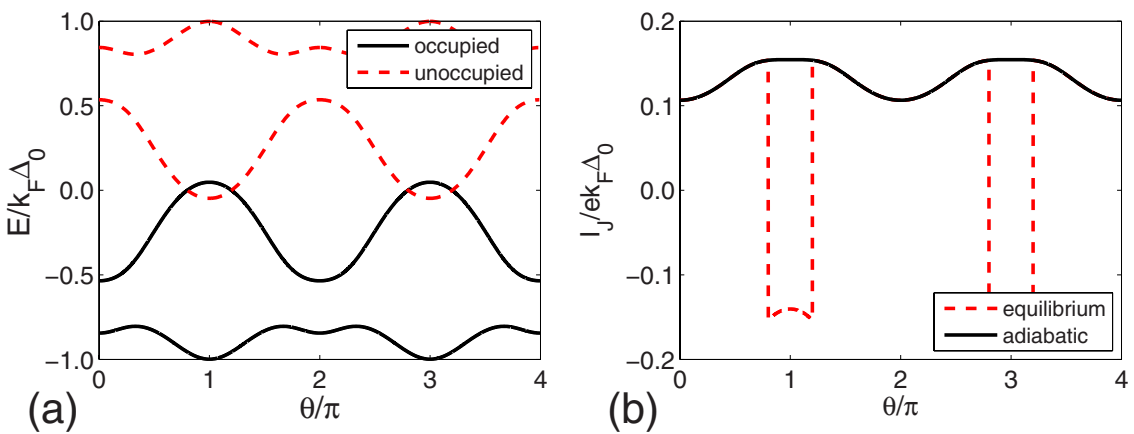

FIG. 13. (Color online) (a) Occupied and unoccupied Andreev states during the adiabatic rotation of $\boldsymbol{d}_{R}$. We fix $g=1.0, g^{\prime}=Z=0, \phi$ $=\pi / 5$, and $\alpha=0$. (b) Josephson current as a function of $\theta$ for rotations in the equilibrium and adiabatic limits. Parameters are the same as in (a).

sal of $I_{J}$ at $\phi=\pi / 5$ with increasing $\theta$ implies that there must be an intermediate state where $I_{J}=0$ for $\phi_{n-1,+}<\phi<\phi_{n,-}$ [the reverse of the situation shown in Fig. 10(a), where $I_{J}$ $=0$ for $\left.\phi_{n,-}<\phi<\phi_{n,+}\right]$. This is indeed observed in Fig. 12(a), where the current for $\phi_{n-1,+}<\phi<\phi_{n,-}$ vanishes at $\theta$ $\approx 0.715 \pi$. When $g^{\prime}$ and $Z$ are both finite, however, $I_{J}$ is not antisymmetric about $\phi=n \pi$, as shown in Fig. 12(b). The sign change in Fig. 11(d) with increasing $\theta$ is therefore accomplished by a downward shift in the value of the current for $\phi_{n-1,+}<\phi<\phi_{n,-}$ : for $0.648 \pi<\theta<\pi$, the current is negative for this range of $\phi$ values. Despite these differences, the presence of a finite $Z$ is apparently a prerequisite: exhaustive numerical investigations for $Z=0$ have failed to find an example where the sign of $I_{J}$ does not reverse across the discontinuity.

\section{Rotating the d vectors: Adiabatic limit}

The adiabatic limit is reached when the rotation period of $\boldsymbol{d}_{R}$ is much faster than the relaxation time of the system, i.e., $T_{\theta} \ll \tau$. As was argued in Ref. 24, the occupations of the Andreev states do not in this case assume their equilibrium values throughout the entire rotation, but rather remain fixed at their initial levels. This implies that the discontinuous jumps in current found in the equilibrium limit due to the zero crossings are absent: as displayed in Fig. 13(a), starting from an initial state at $\theta=0$, the $-a$ level remains fully occupied for all $\theta$, even when it is higher in energy than the $+a$ level for $\theta_{n-}<\theta<\theta_{n+}$. Consequently, the current is a continuous function of $\theta$ and there is no change in the sign of $I_{J}$ during the rotation. This is compared to the result in the equilibrium limit in Fig. 13(b).

A reversal of the Josephson current as $\boldsymbol{d}_{R}$ is rotated is still possible in the adiabatic limit, although this requires a finite potential barrier $Z$ and is not related to the zero-energy crossings of the Andreev states. As seen in Fig. 11(b) for $Z=1.0$ and $\alpha=\pi / 4$, the Josephson current changes sign before the discontinuous jump is reached. As such, starting at $\theta=0$ and rotating $\boldsymbol{d}_{R}$ adiabatically, we therefore encounter a reversal of $I_{J}$ at $\theta \approx 0.6 \pi$. The current changes back to its initial direction at $\theta \approx 1.1 \pi$ (not shown).

\section{Critical current}

The critical current $I_{J c}$ is defined as the maximum magnitude of the current that can be carried by the Josephson junc- tion. The maximum with respect to $\phi$ can occur at three places: a stationary point of $I_{J}$, or on either side of the jump discontinuity at $\phi_{n, \pm}$ (i.e., at $\phi_{n,+} \pm 0^{+}$or equivalently $\left.\phi_{n,-} \mp 0^{+}\right)$. These three critical current locations may be thought of as defining "phases" of the junction. As the barrier parameters or the relative orientations between the two $\boldsymbol{d}$ vectors are varied, we find "transitions" between the different phases. An example of this is shown in Fig. 9(d): as $Z$ or $g^{\prime}$ is increased from zero, the position of $I_{J c}$ changes from $\phi_{n,-}+0^{+}$to the stationary point. The phase boundaries are marked by lines of nonanalyticity in $I_{J C}$. For simplicity, below we specialize to the case $g^{\prime}=0$.

In Fig. 14(a) we plot $I_{J c}$ in the $Z-g$ plane for fixed $\theta$ $=0.35 \pi$ and $\alpha=0.2 \pi$. A line of first-order nonanalyticity, $g_{c 1}(Z)$, is shown as the solid white line; the broken white line shows a line of third-order nonanalyticity at $g_{c 2}(Z)<g_{c 1}(Z)$. The line $g_{c 2}$ defines the boundary between the phase where $I_{J c}$ is located at $\phi=\phi_{n-}-0^{+}\left(g<g_{c 2}\right)$, and the phase where the maximum is located at the stationary point $\left(g_{c 2}<g\right.$ $\left.<g_{c 1}\right)$. For $g>g_{c 1}$, the current maximum is always located at $\phi=\phi_{n-}+0^{+}$. As can be seen in Fig. 14(b), the line of thirdorder nonanalyticity $g_{c 2}$ is not easily detectable in a plot of $I_{J C}$ as a function of $g$; as such, below we consider only lines of first-order nonanalyticity.

We plot the line of first-order nonanalyticity in the Z-g plane for different values of $\theta$ in Fig. 14(c). For orthogonal $\boldsymbol{d}$ vectors (i.e., $\theta=\pi / 2$ ) and $\alpha=\pi / 5$, we do not find any nonanalyticity in the current [since only the squares of $Z$ and $g$ enter into Eq. (37), we need only consider the case $Z, g$ $\geqslant 0]$. Upon an infinitesimal decrease in the value of $\theta$, a line of first-order nonanalyticity appears at $g=0^{+}$; on further decreasing $\theta$, we find that this line moves to higher values of $g$. As $\theta \rightarrow 0$, the line moves to infinity. In contrast, for $Z, g$ $\leqslant 2$ the $Z$ - $g$ plane remains free of first-order nonanalyticities as $\theta$ is increased from $\pi / 2$ to $\pi$ : we have not found any first-order nonanalyticities up to $Z, g=10$, beyond which it becomes difficult to numerically check for nonanalytic behavior. This curious dependence upon $\theta$ can be better appreciated by examining the line of first-order nonanalyticity in the $\alpha-g$ plane [Fig. 14(d)]. We find here an interesting symmetry about $\alpha=0$ : our numerical investigations indicate that the line of nonanalyticity for a $\boldsymbol{d}_{R}$ vector orientation $\pi / 2$ $<\theta<\pi$ can be found by reflecting the line of nonanalyticity for $\boldsymbol{d}_{R}$ vector orientation $\pi-\theta$ about $\alpha=0$. In the limit $\theta$ 


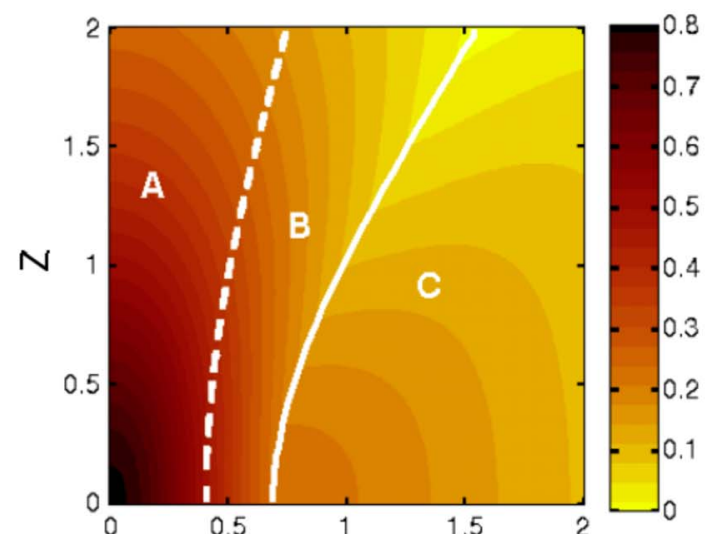

(a)

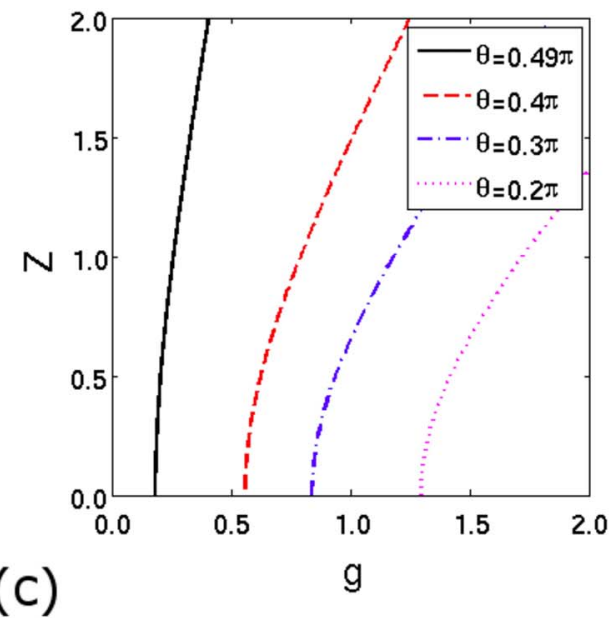

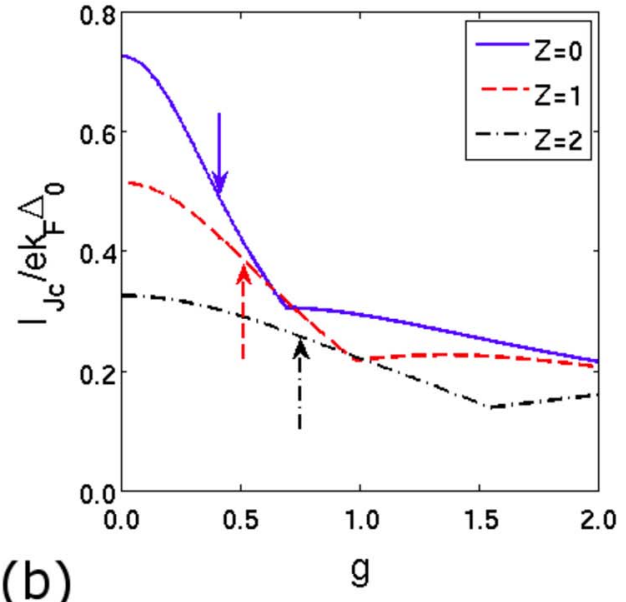

(b)

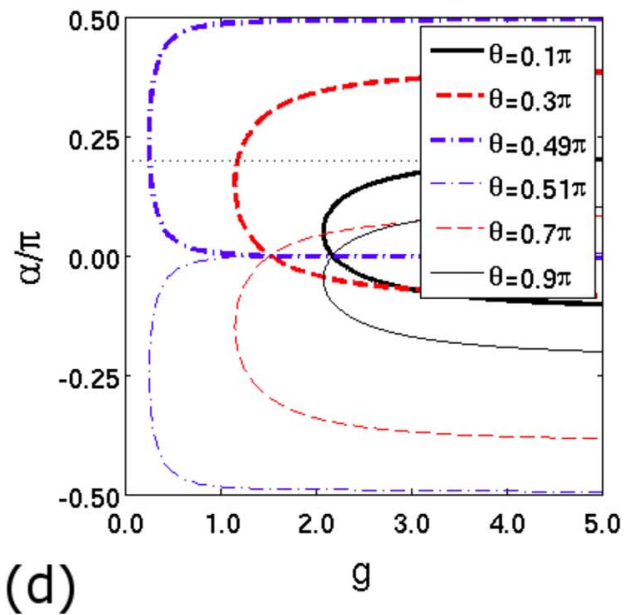

FIG. 14. (Color online) (a) Critical current as a function of $Z$ and $g$ for fixed $\theta=0.35 \pi$ and $\alpha=0.2 \pi$. The value of $I_{J_{C}} / e k_{F} \Delta_{0}$ is given by the scale on the right. The white lines denote boundaries between the different phases of the junction: $I_{J c}$ occurs at $\phi_{n, \pm} \pm 0^{+}$in phase A, at the stationary point in phase $\mathrm{B}$, and in phase $\mathrm{C}$ it occurs at $\phi_{n, \pm} \mp 0^{+}$. The solid line indicates a first-order nonanalyticity in $I_{J c}$; the broken line indicates a third-order nonanalyticity. (b) Critical current as a function of $g$ for fixed $Z$. The point of first-order nonanalyticity is clear as the sharp kink; the position of the third-order nonanalyticity is indicated by the arrows. $\theta$ and $\alpha$ are as in (a). (c) Lines of first-order nonanalyticity of $I_{J C}$ in the $Z$ - $g$ plane for $\alpha=\pi / 5$ and various values of $\theta$. (d) Lines of first-order nonanalyticity of $I_{J C}$ in the $\alpha$ - $g$ plane for $Z=1.0$ and various values of $\theta$.

$=(2 n+1) \pi / 2$, the lines of nonanalyticity are found at constant $\alpha=n \pi / 2$. We have also numerically checked up to high accuracy that the curves in the $\alpha$ - $g$ plane are symmetric about the line $\alpha=\theta / 2(\alpha=\pi / 2-\theta / 2)$ for $0<\theta<\pi / 2(\pi / 2$ $<\theta<\pi)$. Note that the plot in the $\alpha-g$ plane is periodic in $\alpha$ with period $\pi$. The constant line $\alpha=\pi / 5$ is drawn in Fig. 14(d) as the thin dotted line; this corresponds to the situation in Figs. 14(a)-14(c). We see that, as $\theta$ is increased from zero to $\pi / 2$, the intersections of the lines of nonanalyticity with $\alpha=\pi / 5$ occur at progressively smaller values of $g$, consistent with Fig. 14(c). As we cross to the $\pi / 2<\theta<\pi$ case, we see that in the range $0<g<5$ there is no intersection of the lines of nonanalyticity with $\alpha=\pi / 5$. Thus, the absence of nonanalyticity in Fig. 14(c) for $\pi / 2<\theta<\pi$ can be explained by the $\alpha$ dependence shown in Fig. 14(d).

In Fig. 15(a) we plot $I_{J c}$ as a function of $\theta$ and $g$, with fixed $Z=0.5$ and $\alpha=0.3 \pi$. The line of first-order nonanalyticity is again shown by the solid white line, while the third- order nonanalyticities are indicated by the broken white lines. Note the different phases on either side of the firstorder nonanalyticity as compared to Fig. 14(a). In general, a line of first-order nonanalyticity can separate any combination of different phases. The critical current as a function of $\theta$ is shown at several values of fixed $g$ in Fig. 15(b). Although the first-order nonanalyticity is clearly evident as the abrupt change in the slope of $I_{J c}$, the third-order nonanalyticities do not clearly coincide with any noticeable feature.

The line of first-order nonanalyticity in the $\theta-g$ plane displays a complicated variation with $\alpha$ as shown in Fig. 15(c). We see that the limit $\alpha=n \pi$ is somewhat pathological, as here we have a line of nonanalyticity at $\theta=\pi / 2$ which branches into three lines at $g \approx 1.2$, with the top and bottom branches, respectively, converging to $\theta=\pi$ and $\theta=0$ as $g$ $\rightarrow \infty$. For finite $\alpha \neq \alpha_{c}$, the intersection of the branches disappears and the lines of first-order nonanalyticity for $\pi / 2$ $<\theta<\pi$ and $0<\theta \leqslant \pi / 2$ are unconnected. The $\pi / 2<\theta<\pi$ 

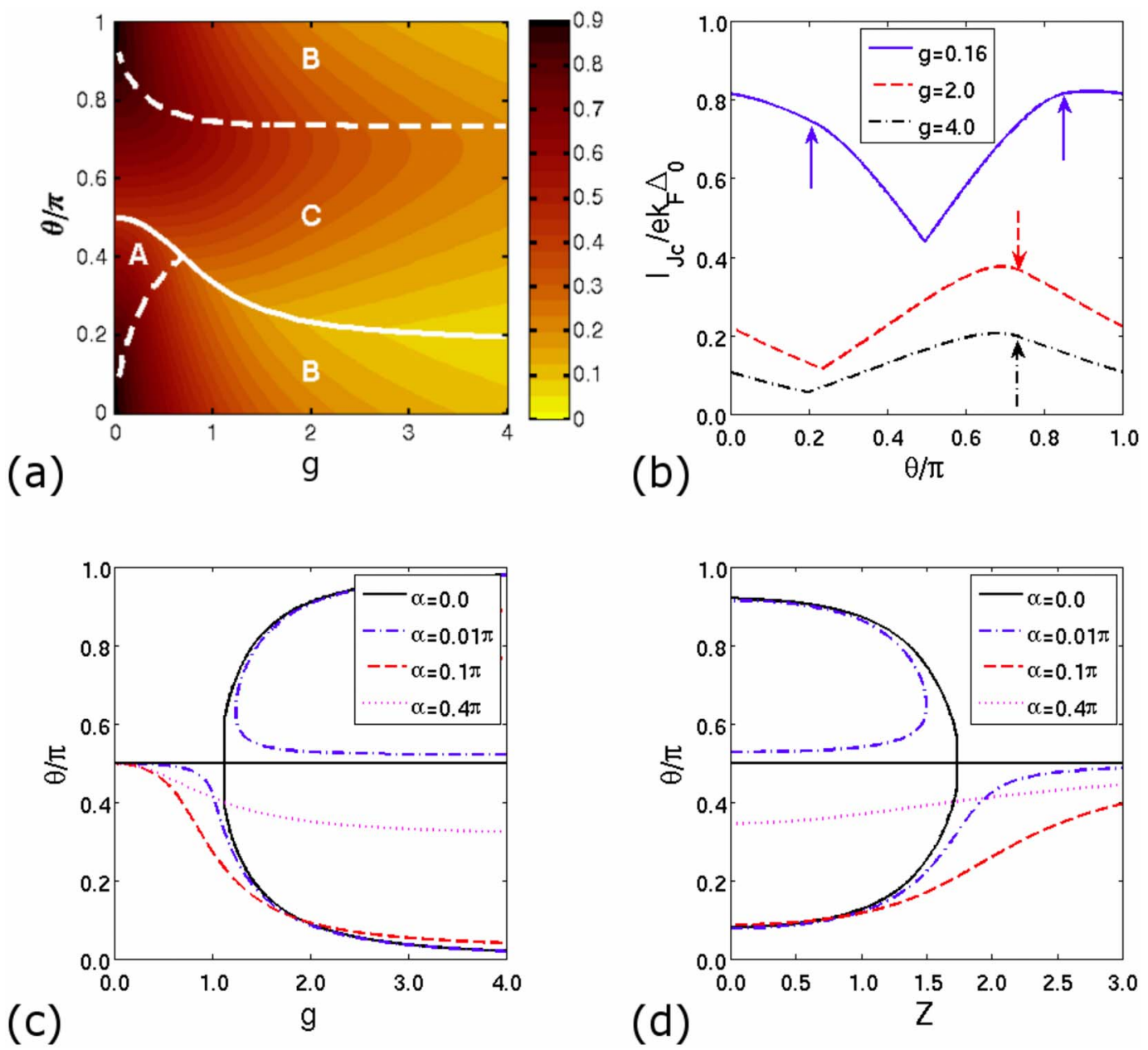

FIG. 15. (Color online) (a) Critical current as a function of $\theta$ and $g$ for $Z=0.5$ and $\alpha=0.3 \pi$. The value of $I_{J c} / e k_{F} \Delta_{0}$ is given by the scale on the right. The white lines denote boundaries between the different phases of the junction: $I_{J c}$ occurs at $\phi_{n, \pm} \pm 0^{+}$in phase A, at the stationary point in phase $\mathrm{B}$, and in phase $\mathrm{C}$ it occurs at $\phi_{n, \pm} \mp 0^{+}$. The solid line indicates a first-order nonanalyticity in $I_{J c}$; the broken lines indicate third-order nonanalyticities. (b) Critical current as a function of $\theta$ for fixed $g$. The point of first-order nonanalyticity is clear as the sharp kink; the third-order nonanalyticities are indicated by the arrows. $Z$ and $\alpha$ are as in (a). (c) Lines of first-order nonanalyticity of $I_{J c}$ in the $\theta-g$ plane for $Z=0.5$ and various values of $\alpha$. (d) Lines of first-order nonanalyticity of $I_{J c}$ in the $\theta-Z$ plane for $g=2.0$ and various values of $\alpha$.

branch disappears as $\alpha$ is increased, while the $g \rightarrow \infty$ limit of the bottom branch moves up to $\theta=\pi / 2$. At $\alpha=\alpha_{c}$, a line of first-order nonanalyticity is found only at constant $\theta=\pi / 2$. Interestingly, this is also the line of nonanalyticity when transverse magnetic terms are absent in the barrier. This can be seen by examining the lines of nonanalyticity in the $\theta-Z$ plane for fixed $g=2.0$, as shown in Fig. 15(d). In the limit $Z / g \rightarrow \infty$, the potential terms in the barrier Hamiltonian dominate the transverse magnetic terms: we accordingly find that, for any value of $\alpha$, the $0<\theta \leqslant \pi / 2$ branch of nonanalyticity asymptotes to $\theta=\pi / 2$ as $Z \rightarrow \infty$. The $\pi / 2<\theta<\pi$ branch, in contrast, is always suppressed beyond some finite $Z$.

\section{CONCLUSIONS}

In this work we have have presented an extended analysis of the novel TFT junction first considered in Ref. 24. Our aim has been twofold: by including a potential scattering term or a component of the magnetization normal to the barrier interface, we have attempted to assess the persistence of the many new effects identified in Ref. 24 under a more general description of the system; we have also investigated the possibility that the presence of these extra terms in the barrier Hamiltonian gives rise to the emergence of other unconventional Josephson behavior, absent in the previously studied case.

We predict that the additional degrees of freedom in the description of the barrier cause three new effects at $T=0$ : the spontaneous generation of a Josephson current by misalignment of the $\boldsymbol{d}$ vectors, even when there is no phase difference between the condensates; the existence of a special line in the parameter space such that $I_{J}$ vanishes over a finite range of $\phi$; and the possibility that the current through a magnetic barrier with potential scattering can show a strong enhancement over its value in the absence of a potential term, no matter what is the orientation of the ferromagnetic moment. 
The $z$ component of the spin current across the junction was calculated for the case $g=0$, and it was found that it could be finite even when the charge current is vanishing. Furthermore, the critical current through the TFT junction was studied as a function of the barrier parameters and $\boldsymbol{d}$-vector alignment. The location of the critical current along the $I_{J}$ vs $\phi$ curves was classified into three qualitatively distinct categories. This enabled the construction of phase diagrams for the TFT junction, where the different phases correspond to different locations of the current maximum. In certain cases a first-order nonanalyticity in the critical current is found at the phase boundaries; we propose using these nonanalyticities as a test of our theoretical knowledge of the TFT junction. Our results also indicate that the current switches identified in Ref. 24 are largely robust to these alterations in the properties of the barrier. Even when $g^{\prime}$ and $Z$ are comparable in magnitude to $g$, we find that it is still possible to use the transverse component of the barrier magnetization to tune the system between off $\left(I_{J} \approx 0\right)$ and on $\left(I_{J} \neq 0\right)$ current states, or to create abrupt reversals in the current direction by small changes in the relative orientation of the $\boldsymbol{d}$ vectors. On the other hand, because of the sensitive dependence of $I_{J}$ upon the $\phi$ dependence of the Andreev states, the reversal of the current with increasing temperature ${ }^{24}$ is strongly suppressed by a finite $Z$ or $g^{\prime}$.

A natural question concerns the observability of the effects predicted above. We first note that our analysis is most applicable to systems where the BdG description of the quasiparticle excitations in the superconducting state is expected to be good. This should be the case for $\mathrm{Sr}_{2} \mathrm{RuO}_{4}$, where the superconducting state is well described by the Balian and Werthamer generalization of the BCS theory to triplet pairing. ${ }^{3,6,7}$ The application to the $p$-wave state in heavyfermion or organic superconductors is less certain, as it is not clear to what extent the standard weak-coupling description of the quasiparticle states is reasonable in these materials. ${ }^{39,40}$

More difficult is the question of experimental control over the different junction parameters. Although it is impossible to forecast future developments in the fabrication of quantum devices, we expect that the orientation of the barrier magnetic moment and the phase difference $\phi$ should be the easiest parameters to manipulate. Much more challenging would be the orientation of the $\boldsymbol{d}$ vectors: as these point in a fixed direction in the crystal, the experimentally accessible values of $\theta$ would be limited by the different orientations in which one could grow a $\mathrm{Sr}_{2} \mathrm{RuO}_{4}$ crystal upon the substrate provided by the magnetic barrier. The increasing degree of control in growing oriented crystal interfaces for tunneling experiments in cuprate superconductors makes us hopeful that this is not an insurmountable obstacle to the experimental study of the $\theta$ dependence of the current. ${ }^{16}$ Alternatively, one could imagine a purely mechanical control over one of the superconducting slabs: indeed, there has recently been much interest in integrating mechanical degrees of freedom into superconducting tunneling experiments, albeit thus far in terms of "charge shuttle" effects. ${ }^{41}$ In any case, we emphasize that the experimental realization of the device proposed here would provide important insights into the interdependence of ferromagnetism and triplet superconductivity.

\section{ACKNOWLEDGMENTS}

The authors are grateful to K. Bennemann, M. L. Kulić, and M. Sigrist for stimulating discussions. J. Sirker is thanked for his critical reading of the manuscript. P.M.R.B. gratefully acknowledges H.-U. Habermeier for facilitating his stay at the Max-Planck-Institut, and thanks A. Simon for his hospitality. B.K. acknowledges support by DLR (German Aerospace Center). D.K.M. acknowledges financial support by the Alexander von Humboldt Foundation, the National Science Foundation under Grant No. DMR-0513415, and the U.S. Department of Energy under Grant No. DE-FG0205ER46225.

\section{APPENDIX A: BOGOLIUBOV TRANSFORMATION AND BOGOLIUBOV-DE GENNES EQUATIONS}

In this appendix, we outline the Bogoliubov-de Gennes transformation to diagonalize the matrix Eq. (11). We introduce the unitary transformation

$$
\begin{gathered}
\Psi(z)=\sum_{n} \hat{A}_{n}(z) \Omega_{n}, \\
\Omega_{n}=\int d z \hat{A}_{n}^{+}(z) \Psi(z),
\end{gathered}
$$

where

$$
\Omega_{n}=\left(\alpha_{n}, \alpha_{n}^{\dagger}, \beta_{n}, \beta_{n}^{\dagger}\right)^{T}
$$

and

$$
\hat{A}_{n}(z)=\left(\begin{array}{cccc}
u_{\alpha, n}(z) & v_{\alpha, n}^{*}(z) & u_{\beta, n}(z) & v_{\beta, n}^{*}(z) \\
v_{\alpha, n}(z) & u_{\alpha, n}^{*}(z) & v_{\beta, n}(z) & u_{\beta, n}^{*}(z) \\
w_{\alpha, n}(z) & x_{\alpha, n}^{*}(z) & w_{\beta, n}(z) & x_{\beta, n}^{*}(z) \\
x_{\alpha, n}(z) & w_{\alpha, n}^{*}(z) & x_{\beta, n}(z) & w_{\beta, n}^{*}(z)
\end{array}\right),
$$

and $\hat{A}_{n}^{+}(z)$ is the Hermitian conjugate of $\hat{A}_{n}(z)$. The diagonalizing spinors $\Omega_{n}$ obey the matrix anticommutation relations

$$
\left\{\Omega_{n}, \Omega_{m}^{\dagger}\right\}=\delta_{m n} \hat{1} .
$$

From the anticommutation relations Eqs. (9) and (A5) we obtain the orthogonality relations for the matrix $\hat{A}_{m}(z)$,

$$
\begin{gathered}
\int d z \hat{A}_{m}^{+}(z) \hat{A}_{n}(z)=\int d z \hat{A}_{m}(z) \hat{A}_{n}^{+}(z)=\delta_{m n} \hat{1}, \\
\sum_{n} \hat{A}_{n}(z) \hat{A}_{n}^{+}\left(z^{\prime}\right)=\sum_{n} \hat{A}_{n}^{+}(z) \hat{A}_{n}\left(z^{\prime}\right)=\delta\left(z-z^{\prime}\right) \hat{1} .
\end{gathered}
$$

Using Eq. (A1) we rewrite the Hamiltonian in terms of the $\Omega_{n}$,

$$
\begin{aligned}
H & =\frac{1}{2} \int d z^{\prime} d z \Psi^{\dagger}\left(z^{\prime}\right) \hat{\mathcal{H}}\left(z^{\prime}, z\right) \Psi(z) \\
& =\frac{1}{2} \sum_{m, n} \Omega_{m}^{\dagger}\left(\int d z^{\prime} d z \hat{A}_{m}^{+}\left(z^{\prime}\right) \hat{\mathcal{H}}\left(z^{\prime}, z\right) \hat{A}_{n}(z)\right) \Omega_{n} .
\end{aligned}
$$

Since the $\hat{A}_{m}(z)$ are assumed to diagonalize $\hat{\mathcal{H}}\left(z^{\prime}, z\right)$, we require that 


$$
\int d z^{\prime} d z \hat{A}_{m}^{+}\left(z^{\prime}\right) \hat{H}\left(z^{\prime}, z\right) \hat{A}_{n}(z)=\hat{E}_{n} \delta_{n m},
$$

where $\hat{E}_{n}$ is a diagonal real matrix. Using the orthogonality condition Eq. (A7), after some algebra we obtain the most general form of the $\mathrm{BdG}$ equations ${ }^{33}$

$$
\int d z^{\prime} \hat{\mathcal{H}}\left(z, z^{\prime}\right) \hat{A}_{n}\left(z^{\prime}\right)=\hat{A}_{n}(z) \hat{E}_{n} .
$$

From the definition of $\hat{A}_{n}(z)$, we may recast this expression as the more familiar eigenvalue problem. Defining the vector of $c$ numbers

$$
\Phi_{j, n}(z)=\left(u_{j, n}(z), v_{j, n}(z), w_{j, n}(z), x_{j, n}(z)\right)^{T},
$$

where $j=\alpha, \beta$, we may write the first $(j=\alpha)$ and third $(j$ $=\beta$ ) columns of Eq. (A10) as

$$
\int d z^{\prime} \hat{\mathcal{H}}\left(z, z^{\prime}\right) \Phi_{j, n}\left(z^{\prime}\right)=E_{j, n} \Phi_{j, n}(z) .
$$

The second and fourth columns of Eq. (A10) are obtained from these by complex conjugation. To obtain Eq. (17), we suppress the $\alpha$ and $\beta$ subscripts in Eq. (A12), as the equations for $j=\alpha$ and $\beta$ are identical. Since we are interested only in the energies of the Andreev bound states (i.e., $n=0$ ), we also drop the subscript $n$, assuming henceforth that we are referring only to the $n=0$ states. That is, we make the replacements $E_{j, n} \rightarrow E, \Phi_{j, n}(z) \rightarrow \Phi(z)$ in Eq. (A12).

\section{APPENDIX B: ANDREEV BOUND STATE ENERGIES}

Here we sketch the derivation of the Andreev bound state energies. Imposing the boundary conditions (19) and (20) on the ansatz (22) results in eight linear relations for the 16 coefficients $u_{\nu, \pm}, v_{\nu, \pm}, w_{\nu, \pm}$, and $x_{\nu, \pm}$. Another eight relations are provided by Eqs. (30) and (31). As in Sec. II B, we assume that $\kappa_{\nu}, 2 m_{\nu} \Delta_{\nu} \hbar^{2} \ll k_{\nu}$ and therefore we may approximate $p_{\nu} \approx k_{\nu}$. The resulting system of 16 homogeneous linear equations has a nontrivial solution only if it is singular, which happens only for certain values of the energy. The explicit condition for these energies is (writing the equations as a matrix equation, the left-hand side of the following equation is, up to an arbitrary nonzero factor, the determinant of the matrix)

$$
\begin{aligned}
\frac{1}{8}[3+ & 3 \cos 2 \gamma_{L} \cos 2 \gamma_{R}-\cos 2 \gamma_{L}-\cos 2 \gamma_{R}+4 \cos 2 \phi \\
& \left.+4 \cos 2\left(\bar{\theta}_{L}-\bar{\theta}_{R}\right)+16 \cos \left(\bar{\theta}_{L}-\bar{\theta}_{R}\right) \sin \gamma_{L} \sin \gamma_{R} \cos \phi\right] \\
& -8 Z g^{\prime} \cos \gamma_{L} \cos \gamma_{R} \sin \left(\bar{\theta}_{L}-\bar{\theta}_{R}\right) \sin \phi+\left(r+r^{-1}\right)\left\{2 \left(Z^{2}\right.\right.
\end{aligned}
$$

$$
\begin{aligned}
& \left.+g^{\prime 2}\right) \cos ^{2} \gamma_{L} \cos ^{2} \gamma_{R}-\cos \gamma_{L} \cos \gamma_{R}\left[\cos \left(\bar{\theta}_{L}-\bar{\theta}_{R}\right) \cos \phi\right. \\
& \left.\left.+\sin \gamma_{L} \sin \gamma_{R}\right]\right\}+\frac{1}{4}\left(r^{2}+r^{-2}\right) \cos ^{2} \gamma_{L} \cos ^{2} \gamma_{R}+4\left(Z^{2}\right. \\
& \left.-g^{\prime 2}\right)^{2} \cos ^{2} \gamma_{L} \cos ^{2} \gamma_{R}-4\left(Z^{2}\right. \\
& \left.+g^{\prime 2}\right) \cos \gamma_{L} \cos \gamma_{R}\left[\cos \left(\bar{\theta}_{L}-\bar{\theta}_{R}\right) \cos \phi+\sin \gamma_{L} \sin \gamma_{R}\right] \\
& -2 g^{2}\left\{4\left(Z^{2}-g^{\prime 2}\right) \cos ^{2} \gamma_{L} \cos ^{2} \gamma_{R}\right. \\
& -2 \cos \gamma_{L} \cos \gamma_{R}\left[\cos \left(\bar{\theta}_{L}+\bar{\theta}_{R}\right) \cos \phi-\sin \gamma_{L} \sin \gamma_{R}\right] \\
& +r \cos \gamma_{R}\left(\sin ^{2} \gamma_{L}+\cos 2 \bar{\theta}_{L}\right)+r^{-1} \cos ^{2} \gamma_{L}\left(\sin ^{2} \gamma_{R}\right. \\
& \left.\left.+\cos 2 \bar{\theta}_{R}\right)\right\}+4 g^{4} \cos ^{2} \gamma_{L} \cos ^{2} \gamma_{R}=0,
\end{aligned}
$$

where we have adopted the notations $r=k_{L} / k_{R}, \bar{\theta}_{L}=\theta_{L}-\alpha$, $\bar{\theta}_{R}=\theta_{R}-\alpha$, and define $\gamma_{\nu}$ by

$$
\cos \gamma_{\nu}=\frac{E}{k_{\nu} \Delta_{\nu}}, \quad \sin \gamma_{\nu}=\frac{\hbar^{2} \kappa_{\nu}}{m_{\nu} \Delta_{\nu}} .
$$

The energies $E$ of the Andreev states follow from the solution of Eq. (B1) together with the definitions of $\gamma_{\nu}$ [Eq. (B2)] and $\kappa_{\nu}$ [Eq. (32)]. As such, they must respect the underlying symmetries of the Hamiltonian. In particular, we note that the $E$ depend on $\theta_{L}, \theta_{R}$, and $\alpha$ only through the differences $\bar{\theta}_{L}=\theta_{L}-\alpha$ and $\bar{\theta}_{R}=\theta_{R}-\alpha$, as dictated by rotation invariance around the $z$ axis. Without loss of generality, we therefore set $\theta_{L}=0$ and $\theta_{R}=\theta$, i.e., the direction of $\boldsymbol{d}_{L}$ defines the $x$ axis. Furthermore, we see that the solutions of Eq. (B1) are invariant under $(g, \alpha) \rightarrow(-g, \alpha \pm \pi)$, as expected from the definition of $\mathcal{H}_{\perp}$ in Eq. (4). Equation (B1) also provides a general condition for the existence of zero-energy solutions. When $E=0$ we have from Eq. (B2) the identity $\cos \gamma_{R}=\cos \gamma_{L}=0$; Eq. (B1) then reduces to

$$
\cos \frac{1}{2}(\theta-\phi) \cos \frac{1}{2}(\theta+\phi)=0 .
$$

Thus there is a zero-energy solution whenever $\cos \frac{1}{2}(\theta \pm \phi)$ $=0$. This condition is independent of the value of $r$ and the details of the scattering potential at the barrier. When the superconductors on either side of the gap are made from the same material, i.e., we have $k_{L}=k_{R} \equiv k_{F}, m_{L}=m_{R}$, and $k_{L} \Delta_{L}$ $=k_{R} \Delta_{R} \equiv k_{F} \Delta_{0}$, Eq. (B1) reduces to a quadratic equation in $E^{2}$

$$
\frac{E^{4}}{D^{2} k_{F}^{4} \Delta_{0}^{4}}-4 A \frac{E^{2}}{k_{F}^{2} \Delta_{0}^{2}}+4 B^{2}=0,
$$

where $A, B$ and $D$ are defined in Eqs. (34)-(36), respectively. Equation (B4) is solved by the bound state energies (33). 
${ }^{1}$ D. J. Scalapino, Phys. Rep. 250, 329 (1995); D. J. van Harlingen, Rev. Mod. Phys. 67, 515 (1995); C. C. Tsuei and J. R. Kirtley, ibid. 72, 969 (2000).

${ }^{2}$ J. F. Annett, Physica C 317-318, 1 (1999).

${ }^{3}$ R. Balian and N. R. Werthamer, Phys. Rev. 131, 1553 (1963).

${ }^{4}$ Y. Maeno, H. Hashimoto, K. Yoshida, S. Nishizaki, T. Fujita, J. G. Bednorz, and F. Y. Lichtenberg, Nature (London) 372, 532 (1994).

${ }^{5}$ A. P. Mackenzie and Y. Maeno, Rev. Mod. Phys. 75, 657 (2003).

${ }^{6}$ T. M. Rice and M. Sigrist, J. Phys.: Condens. Matter 7, L643 (1995)

${ }^{7}$ M. Sigrist, D. Agterberg, A. Furusaki, C. Honerkamp, K. K. Ng, T. M. Rice, and M. E. Zhitomirsky, Physica C 317, 134 (1999).

${ }^{8}$ J. P. Brison, L. Glemot, H. Suderow, A. Huxley, S. Kambe, and J. Flouquet, Physica B 280, 165 (2000).

${ }^{9}$ I. J. Lee, S. E. Brown, W. G. Clark, M. J. Strouse, M. J. Naughton, W. Kang, and P. M. Chaikin, Phys. Rev. Lett. 88, 017004 (2001); I. J. Lee, D. S. Chow, W. G. Clark, M. J. Strouse, M. J. Naughton, P. M. Chaikin, and S. E. Brown, Phys. Rev. B 68, 092510 (2003).

${ }^{10}$ S. S. Saxena, P. Agarwal, K. Ahilan, F. M. Grosche, R. K. W. Haselwimmer, M. J. Steiner, E. Pugh, I. R. Walker, S. R. Julian, P. Monthoux, G. G. Lonzarich, A. Huxley, I. Sheikin, D. Braithwaite, and J. Flouquet, Nature (London) 406, 587 (2000); A. Huxley, I. Sheikin, E. Ressouche, N. Kernavanois, D. Braithwaite, R. Calemczuk, and J. Flouquet, Phys. Rev. B 63, 144519 (2001).

${ }^{11}$ D. Aoki, A. Huxley, E. Ressouche, D. Braithwaite, J. Flouquet, J. P. Brison, E. L'hôtel, and C. D. Paulsen, Nature (London) 413, 613 (2001).

${ }^{12}$ Y. Maeno, Physica C 282, 206 (1997).

${ }^{13}$ P. G. de Gennes and D. Saint-James, Phys. Lett. 4, 151 (1963); A. F. Andreev, Sov. Phys. JETP 22, 455 (1966).

${ }^{14}$ A. M. Zagoskin, Quantum Theory of Many-Body Systems (Springer, New York, 1998).

${ }^{15}$ I. O. Kulik, Sov. Phys. JETP 30, 944 (1970); C. Ishii, Prog. Theor. Phys. 44, 1525 (1970).

${ }^{16}$ S. Kashiwaya and Y. Tanaka, Rep. Prog. Phys. 63, 1641 (2000).

${ }^{17}$ Y. S. Barash, H. Burkhardt, and D. Rainer, Phys. Rev. Lett. 77, 4070 (1996); Y. Tanaka and S. Kashiwaya, Phys. Rev. B 53, R11957 (1996); S. K. Yip, J. Low Temp. Phys. 109, 547 (1997); R. A. Riedel and P. F. Bagwell, Phys. Rev. B 57, 6084 (1998).

${ }^{18}$ Y. Asano, Phys. Rev. B 64, 224515 (2001); , J. Phys. Soc. Jpn. 71, 905 (2002).

${ }^{19}$ C. D. Vaccarella, R. D. Duncan, and C. A. R. Sá de Melo, Physica C 391, 89 (2003).

${ }^{20}$ Y. Asano, Y. Tanaka, M. Sigrist, and S. Kashiwaya, Phys. Rev. B 67, 184505 (2003).
${ }^{21}$ H.-J. Kwon, K. Sengupta, and V. M. Yakovenko, Eur. Phys. J. B 37, 349 (2004).

${ }^{22}$ Y. Asano, Phys. Rev. B 72, 092508 (2005); 74, 220501(R) (2006).

${ }^{23}$ T. Yokoyama, Y. Tanaka, and A. A. Golubov, Phys. Rev. B 75, 094514 (2007); Y. Sawa, T. Yokoyama, Y. Tanaka, and A. A. Golubov, ibid. 75, 134508 (2007).

${ }^{24}$ B. Kastening, D. K. Morr, D. Manske, and K. Bennemann, Phys. Rev. Lett. 96, 047009 (2006).

${ }^{25}$ A. A. Golubov, M. Yu. Kupriyanov, and E. Il'ichev, Rev. Mod. Phys. 76, 411 (2004).

${ }^{26}$ L. N. Bulaevskii, V. V. Kuzii, and A. A. Sobyanin, JETP Lett. 25, 290 (1977); A. V. Andreev, A. I. Buzdin, and R. M. Osgood III, Phys. Rev. B 43, 10124 (1991); A. I. Buzdin, B. Vujicic, and M. Yu. Kupriyanov, Sov. Phys. JETP 74, 124 (1992).

${ }^{27}$ V. V. Ryazanov, V. A. Oboznov, A. Yu. Rusanov, A. V. Veretennikov, A. A. Golubov, and J. Aarts, Phys. Rev. Lett. 86, 2427 (2001).

${ }^{28}$ M. Fogelström, Phys. Rev. B 62, 11812 (2000); M. Andersson, J. C. Cuevas, and M. Fogelström, Physica C 117, 367 (2002); Yu. S. Barash and I. V. Bobkova, Phys. Rev. B 65, 144502 (2002).

${ }^{29}$ B. Kastening, D. K. Morr, L. Alff, and K. Bennemann, arXiv:cond-mat/0610283 (unpublished).

${ }^{30}$ M. S. Grønsleth, J. Linder, J.-M. Børven, and A. Sudb $\varnothing$, Phys. Rev. Lett. 97, 147002 (2006); J. Linder, M. S. Grønsleth, and A. Sudbø, Phys. Rev. B 75, 024508 (2007).

${ }^{31}$ Y. Zhao and R. Shen, Phys. Rev. B 73, 214511 (2006).

${ }^{32}$ B. E. Kane, Nature (London) 393, 133 (1998); L. B. Ioffe, V. B. Geshkenbein, M. V. Feigel'man, A. L. Fauchere, and G. Blatter, ibid. 398, 679 (1999).

${ }^{33}$ P. G. de Gennes, Superconductivity of Metals and Alloys (Addison-Wesley, New York, 1989).

${ }^{34}$ M. L. Kulić and I. M. Kulić, Phys. Rev. B 63, 104503 (2001); I. Eremin, F. S. Nogueira, and R.-J. Tarento, ibid. 73, 054507 (2006).

${ }^{35}$ M. Vuorio, J. Phys. C 9, L267 (1976).

${ }^{36}$ A. S. Borovik-Romanov, Y. M. Bunkov, V. V. Dmitriev, Y. M. Mukharskiy, and D. A. Sergatskov, Phys. Rev. Lett. 62, 1631 (1989).

${ }^{37}$ I. A. Fomin, Physica B 169, 153 (1991).

${ }^{38}$ J. C. Davis and R. E. Packard, Rev. Mod. Phys. 74, 741 (2002).

${ }^{39}$ G. R. Stewart, Rev. Mod. Phys. 73, 797 (2001).

${ }^{40}$ D. Jérome, Chem. Rev. (Washington, D.C.) 104, 5565 (2004).

${ }^{41}$ L. Y. Gorelik, A. Isacsson, Y. M. Galperin, R. I. Shekhter, and M. Jonson, Nature (London) 411, 454 (2001); T. Novotný, A. Donarini, and A.-P. Jauho, Phys. Rev. Lett. 90, 256801 (2003); J. Fransson and A. V. Balatsky, arXiv:0707.0495 (unpublished). 